\title{
Resolution Dependent Relative Dispersion Statistics in a Hierarchy of Ocean Models
}

\author{
Andrew C. Poje ${ }^{\mathrm{a}}$, Angelique C. Haza ${ }^{\mathrm{b}}$, Tamay M. Özgökmen ${ }^{\mathrm{b}}$, Marcello G. Magaldi ${ }^{\mathrm{c}}$, Zulema D. Garraffo ${ }^{\mathrm{d}}$ \\ ${ }^{a}$ Department of Mathematics, College of Staten Island, CUNY, Staten Island, New York. \\ ${ }^{b}$ RSMAS/MPO, University of Miami, Miami, Florida. \\ ${ }^{c}$ Department of Earth and Planetary Sciences, Johns Hopkins University, Baltimore, Maryland. \\ ${ }^{d}$ Center for Computational Science, University of Miami, Miami, Florida.
}

\begin{abstract}
In order to determine the effect of Eulerian spatial resolution on the two particle statistics of synthetic drifter trajectories, we examine a hierarchy of ocean models, starting from 2D turbulence simulations, progressing to idealized simulations of a buoyant coastal jet with ROMS, and finally to realistic HYCOM simulations of the Gulf Stream. In each case, particle dispersion at large time and space scales is found to be controlled by energetic meso-scale features of the flow that are relatively insensitive to the resolution of finer scale motions. In all cases, time-distance graphs given in terms of computed Finite Scale Lyapunov Exponents show an expected increase in the extent of exponential scaling with increasing spatial smoothing of the velocity field. The limiting value of the FSLE at small separation distances is found to scale remarkably well with the resolution of Eulerian velocity gradients as given by the average of positive Okubo-Weiss parameter values.
\end{abstract}

Key words:

\section{Introduction}

An ocean model's ability to accurately predict the complex statistics of the relative dispersion of marked particle pairs is of primary importance for a variety of practical purposes. The study of relative dispersion, directly connected to parameterizations of turbulent mixing and scalar fluctuations, has been the focus of intense modeling and theoretical efforts over the past century (see reviews Sawford (2001); Garrett (2006); LaCasce (2008)).

As pointed out by Griffa et al. (2004), there continues to exist (and will continue to exist) a significant gap between spatial and temporal scales resolved by even the highest resolution ocean model and the scales effecting the motion of Lagrangian particles in the actual ocean. The nonlinear evolution of tracer trajectories given by

$$
\frac{d \mathbf{x}}{d t}=\mathbf{v}(t)=\mathbf{u}(\mathbf{x}, t)
$$

implies that particle-based Lagrangian measures depend upon the integrated effect of all spatial and temporal scales of motion. An essential question is how well necessarily under-resolved ocean models capture the physics of particle dispersion.

Such questions have become increasingly important for a number of reasons. Given recent advances in hardware and algorithms and the increasing availability and assimilation of data sources, coastal and basin scale ocean models are now routinely called upon to address detailed prediction of both passive and active tracer components in realistic settings for biological and environmental purposes. In addition, a number of studies have recently investigated the detailed geometry of mixing and transport in the ocean using dynamical systems techniques that locate distinguished finite-time invariant flow boundaries in the Lagrangian frame (Haller, 2001; Shadden et al., 2005). Whether based upon velocity fields derived from quasi-geostrophic analysis of satellite surface data (Abraham and Bowen, 2002; Waugh et al., 2006; BeronVera et al., 2008)), high-frequency radar signals (Lipphardt et al., 2006; Coulliette et al., 2007) or directly 
computed from models (d'Ovidio et al., 2004; Olascoaga et al., 2006; Haza et al., 2007a; Mancho et al., 2008), deduction of so-called Lagrangian Coherent Structures, by any of the available methods, ultimately requires accurate estimate of two-particle dispersion statistics. The sensitivity of such statistics to the spatial resolution of the input velocity fields ultimately determines the robustness of the resulting transport geometry.

The goal here is to directly investigate the relationship between Eulerian model resolution and the resulting behavior of Lagrangian dispersion statistics. Our approach follows that of Iudicone et al. (2002); Griffa et al. (2004); Bracco et al. (2004); Haza et al. (2008). We consider three distinct flow fields of increasing complexity in which we have varying degrees of control over our ability to change the spatial resolution of the model velocity. We begin with the standard case of statistically stationary and homogeneous two dimensional turbulence and examine the effects of dynamically consistent smoothing on dispersion statistics. This is done by first fully resolving all Fourier modes in the simulation and then considering advection by velocity fields constructed from a smaller number of low wavenumber modes whose phases are determined by the full simulation. We then relax the assumption of homogeneity by examining a baroclinically unstable wall-bounded jet simulated using ROMS. In this case, we study changes in the resulting relative dispersion statistics by directly varying the model spatial resolution for constant values of the input forcing. Finally, we consider a fixed, high resolution HYCOM North Atlantic simulation and examine spatial resolution effects by post-processing the model data with spatial filters of varying scale. In each case, we investigate the relative dispersion using both standard statistics and doubling time (Finite Size Lyapunov) metrics to highlight scale resolution dependence.

\section{Relative dispersion}

We consider the separation statistics of particle pair trajectories given by solutions of Eq. (1). Borrowing standard notation from Babiano et al. (1990) and denoting the trajectory by $\mathbf{x}(\mathbf{a}, t)$ where $\mathbf{x}\left(\mathbf{a}, t_{0}\right)=\mathbf{a}$, the relative separation of a particle pair is given by

$$
\mathbf{D}\left(t, \mathbf{D}_{0}\right)=\mathbf{D}_{0}+\left(\mathbf{x}\left(\mathbf{a}_{1}, t\right)-\mathbf{x}\left(\mathbf{a}_{2}, t\right)\right)=\mathbf{D}_{0}+\int_{t_{0}}^{t} \delta \mathbf{v}\left(t, \mathbf{D}_{0}\right) d t^{\prime}
$$

where the Lagrangian velocity difference is defined by

$$
\delta \mathbf{v}\left(t, \mathbf{D}_{0}\right)=\left(\mathbf{v}\left(\mathbf{a}_{1}, t\right)-\mathbf{v}\left(\mathbf{a}_{2}, t\right)\right) .
$$

The statistical quantities of interest are the relative dispersion,

$$
D^{2}=\langle\mathbf{D} \cdot \mathbf{D}\rangle
$$

and the relative diffusivity

$$
K(t)=\frac{1}{2} \frac{d D^{2}}{d t}=\left\langle\mathbf{D}\left(t, \mathbf{D}_{0}\right) \cdot \delta \mathbf{v}\left(t, \mathbf{D}_{0}\right)\right\rangle
$$

where $\langle\cdot\rangle$ is the average over all particle pairs. In terms of individual Lagrangian velocities, Eq. (4) is equivalent to

$$
K(t)=2 \int_{t_{0}}^{t}\left\langle\mathbf{v}(\mathbf{a}, t) \cdot \mathbf{v}\left(\mathbf{a}, t^{\prime}\right)\right\rangle_{a} d t^{\prime}-2 \int_{t_{0}}^{t}\left\langle\mathbf{v}\left(\mathbf{a}_{1}, t\right) \cdot \mathbf{v}\left(\mathbf{a}_{2}, t^{\prime}\right)\right\rangle d t^{\prime}+\left\langle\mathbf{D}_{0} \cdot \delta \mathbf{v}\left(t, \mathbf{D}_{0}\right)\right\rangle .
$$

The relative diffusivity is then the sum of three contributions. The first term on the right hand side of Eq. (5) is simply twice the absolute diffusivity of individual trajectories given by the single particle Lagrangian velocity auto-correlation, $\langle\cdot\rangle_{a}$ denotes averaging over single particles, not particle pairs. The second term is the cross-correlation of pair velocities. The third term, the correlation between initial pair separation and velocity differences, vanishes in the case of statistically homogeneous flows for randomly distributed particle 
pairs. In inhomogeneous flows, this correlation is expected to decay in time as particles lose memory of their initial state.

The asymptotic behavior of the relative dispersion for small and large times is well established (Batchelor, 1952; Babiano et al., 1990; LaCasce, 2008). For very short times, the velocity difference of particles that are initially close is approximately constant and $D^{2}(t) \sim t^{2}$ while $K(t)$ grows linearly in time. For large times (large separation distances), both the second and third terms in Eq. (5) decay to zero as the velocities along each trajectory become uncorrelated. For flows with finite Lagrangian time scales, $K(t)$ is constant in the long time limit and the asymptotic growth of $D^{2}(t)$ is linear. Similarly, for vanishingly small separations in suitably smooth flow fields, first order expansion implies that

$$
\frac{d \mathbf{D}}{d t} \sim \nabla \mathbf{u} \cdot \mathbf{D}
$$

with $\nabla \mathbf{u}$ independent of separation distance. In this scale-independent regime, $D \sim \mathrm{e}^{\alpha t}$.

At intermediate time and separation scales where two point velocity statistics are correlated, the question of the behavior of the relative dispersion is less clear, especially in geophysical flows where the effects of rotation and stratification typically lead to the formation of energetic coherent vortex structures. For statistically homogeneous flows, Richardson's theory Richardson (1926), $D^{2} \sim t^{3}$, is often observed and is consistent with both the Kolmogorov-Kraichnan inverse energy cascade in 2D turbulence (Batchelor, 1952) and the saturation of two-point Lagrangian-acceleration correlations (Babiano et al., 1990; Castilla et al., 2007). However, the presence of long-lived coherent vortices, as shown by Ehlmaidi et al. (1993), leads to so-called anomalous dispersion regimes with super-diffusive scaling due to preferential sampling of the hyperbolic regimes on the periphery of vortex cores.

Much of our understanding of numerical and observational investigations of relative dispersion statistics in the ocean derives from the limiting case of $2 \mathrm{D}$, homogeneous, isotropic turbulence. The dynamics of 2-D homogeneous turbulence forced at a single scale are well established and consist of two distinct cascade regimes determined by the forcing wavenumber $k_{f}$. A direct cascade of enstrophy to smaller scales exists for scales smaller than $k_{f}^{-1}$ and an inverse energy cascade to larger scales for scales larger than $k_{f}^{-1}$. The enstrophy cascade is typically associated with a steep, nonlocal, slope of the energy spectrum $E(k) \sim k^{-3}$ while Kolmogorov-Kraichnan inertial range arguments in the inverse cascade range imply $E(k) \sim k^{-5 / 3}$.

The implications of the dual cascade on the relative dispersion of particle pairs are also well known. Following Bennett (1984) (see also Castilla et al. (2007); LaCasce (2008)), for a homogeneous and statistically stationary 2-D turbulence, the Lagrangian velocity difference responsible for particle pair separation as given in Eq. (2) is equivalent to the second-order Eulerian structure function, $\left\langle\delta \mathbf{v}^{2}(\mathbf{D})\right\rangle=$ $\overline{(\mathbf{u}(x+\mathbf{D}, t)-\mathbf{u}(x, t))^{2}}=S(\mathbf{D})$. Dimensional arguments can then be used to relate the scaling of the relative dispersion to the local slope of the energy spectrum of the underlying Eulerian velocity field, $E(k)$. Given the assumption

$$
K(D)=\langle\mathbf{D} \cdot \delta \mathbf{v}\rangle \sim S^{1 / 2} D
$$

and the relationship

$$
S(D)=2 \int_{0}^{\infty} E(k)\left(1-J_{0}(k D)\right) d k
$$

standard asymptotics show that for Eulerian spectra with power law dependence, $E(k) \sim k^{-\beta}$,

$$
\begin{array}{rlrl}
S(D) \sim D^{\beta-1}, & K(D) & \sim D^{\beta+1) / 2}, \quad D \sim t^{2 /(3-\beta)}, \quad 1<\beta<3 \\
S(D) \sim D^{2}, & K(D) & \sim D^{2}, \quad D \sim \mathrm{e}^{\alpha t}, \quad \beta>3 . &
\end{array}
$$

The limiting value, $\beta=3$, separates regimes of local dispersion for $\beta<3$ where pair separations are dominated by motions at the local separation scale from the nonlocal regime $\beta>3$ where separation statistics are determined by the dynamics at scales larger than the local separation. Thus, for 2-D turbulence in the enstrophy cascade range one expects exponential behavior of the relative dispersion while Eq. (6) predicts Richardson scaling in the inverse cascade regime if $\beta=5 / 3$. 


\section{2D Turbulence}

As a first example, we consider particle evolution under the action of velocity fields produced by direct numerical solutions of the 2-D vorticity equation

$$
\begin{aligned}
\frac{\partial \omega}{\partial t}+J(\psi, \omega) & =F(\omega)+D(\omega) \\
\omega & =\nabla^{2} \psi
\end{aligned}
$$

where $J(\cdot, \cdot)$ is the two-dimensional Jacobian operator and $F$ and $D$ are forcing and dissipation operators chosen to produce statistically steady vorticity distributions. The forcing term, $F\left(\omega ; k_{f}\right)$ is constructed to insure that the energy in the spectral solution at wavenumber $k_{f}=\left\|\mathbf{k}_{f}\right\|$ is kept constant while the phases of the modes at this wavenumber evolve freely under the dynamics. Energy dissipation, $D$, is the sum of an eighth-order hyperviscocity at small scales and a linear drag at the largest scales,

$$
D(\omega)=\nu_{h}\left(\nabla^{2}\right)^{8} \omega-\alpha \psi
$$

Solutions are computed by a standard spectral solver in a periodic domain of size $2 \pi \times 2 \pi$. In all cases, particle trajectories are computed alongside the Eulerian fields using a spectral spline interpolation scheme in space and a second order Adams-Bashforth scheme in time.
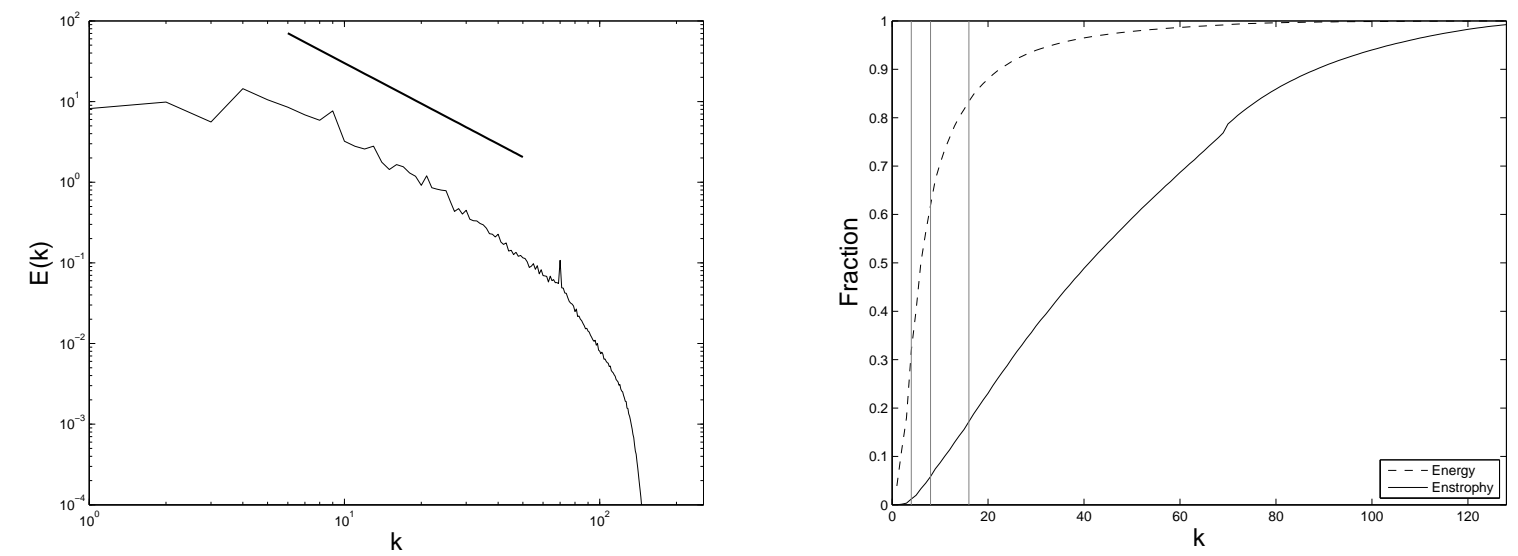

Figure 1: (a) Kinetic energy spectrum for the $k_{f}=70$ simulation. The solid line indicates $-5 / 3$ slope. (b) The fraction of energy and enstrophy resolved as a function of smoothing wavenumber $k_{c}$. Vertical lines indicate resolved fractions at $k_{c}=8$, 16 , and 32 .

For this study we concentrate on a simulation of Eq. (7) forced at $k=70$ with a moderate resolution of $512^{2}$ modes. Specific parameters in the model are given in Table 1. Fig. 1 shows the resulting energy spectrum averaged over $\tau=t\left(\overline{\omega^{2}}\right)^{1 / 2} \sim 1000$ eddy turnover times. Throughout, the energy in the simulation varied by $\sim 1.5 \%$ around about an average of 121 while the enstrophy remained within $\sim 4 \%$ of $\overline{\omega^{2}}=28800$. The simulation produces a distinct inverse cascade regime at scales $\sim 10<k<k_{f} / 2$ followed by a much narrower direct cascade at wavenumbers $>k_{f}$. Also shown in the figure are the cumulative distributions of both energy and enstrophy in the simulation as a function of wavenumber. As expected, the energy is narrowly concentrated at large scales while the enstrophy fraction asymptotes much more slowly. For reference, the first 32 wave-numbers capture more than $80 \%$ of the total energy but less than $25 \%$ of the total enstrophy.

To determine the effect of spatial resolution on Lagrangian dispersion statistics, we consider the following spatial smoothing of the fully resolved Eulerian velocity field. For a given $\psi(\mathbf{k}, t)$ produced by the full simulation of Eq. (7), we define an infinitely steep spectral truncation operator

$$
\hat{\psi}(\mathbf{k}, t)= \begin{cases}\psi(\mathbf{k}, t) & \text { if }|\mathbf{k}| \leq k_{c} \\ 0 & \text { if }|\mathbf{k}|>k_{c}\end{cases}
$$




\begin{tabular}{|c|c|c|c|c|c|}
\hline $\mathrm{N}$ & $k_{f}$ & $\nu_{h}$ & $\alpha$ & Energy & $\overline{\omega^{2}}$ \\
\hline 512 & 70 & $2.5 \times 10^{-31}$ & 2.0 & 121 & 28800 \\
\hline
\end{tabular}

Table 1: Parameters for numerical simulation.

and then examine, as a function of $k_{c}$, statistics of Lagrangian trajectories advected by the truncated velocity field

$$
\frac{d x\left(t ; k_{c}\right)}{d t}=-\frac{\partial \hat{\psi}}{\partial y}, \quad \frac{d y\left(t ; k_{c}\right)}{d t}=\frac{\partial \hat{\psi}}{\partial x}
$$

Since the time dependence of the spatially smooth field, $\hat{\psi}(\mathbf{k}, t)$, is inherited directly from the full solution, the resulting advection fields are dynamically consistent and identical with $\psi(\mathbf{k}, t)$ at spatial scales larger than $2 \pi / k_{c}$. Snapshots of the resulting stream function field with $k_{f}=70$ for various values of $k_{c}$ are shown in Fig. 2.
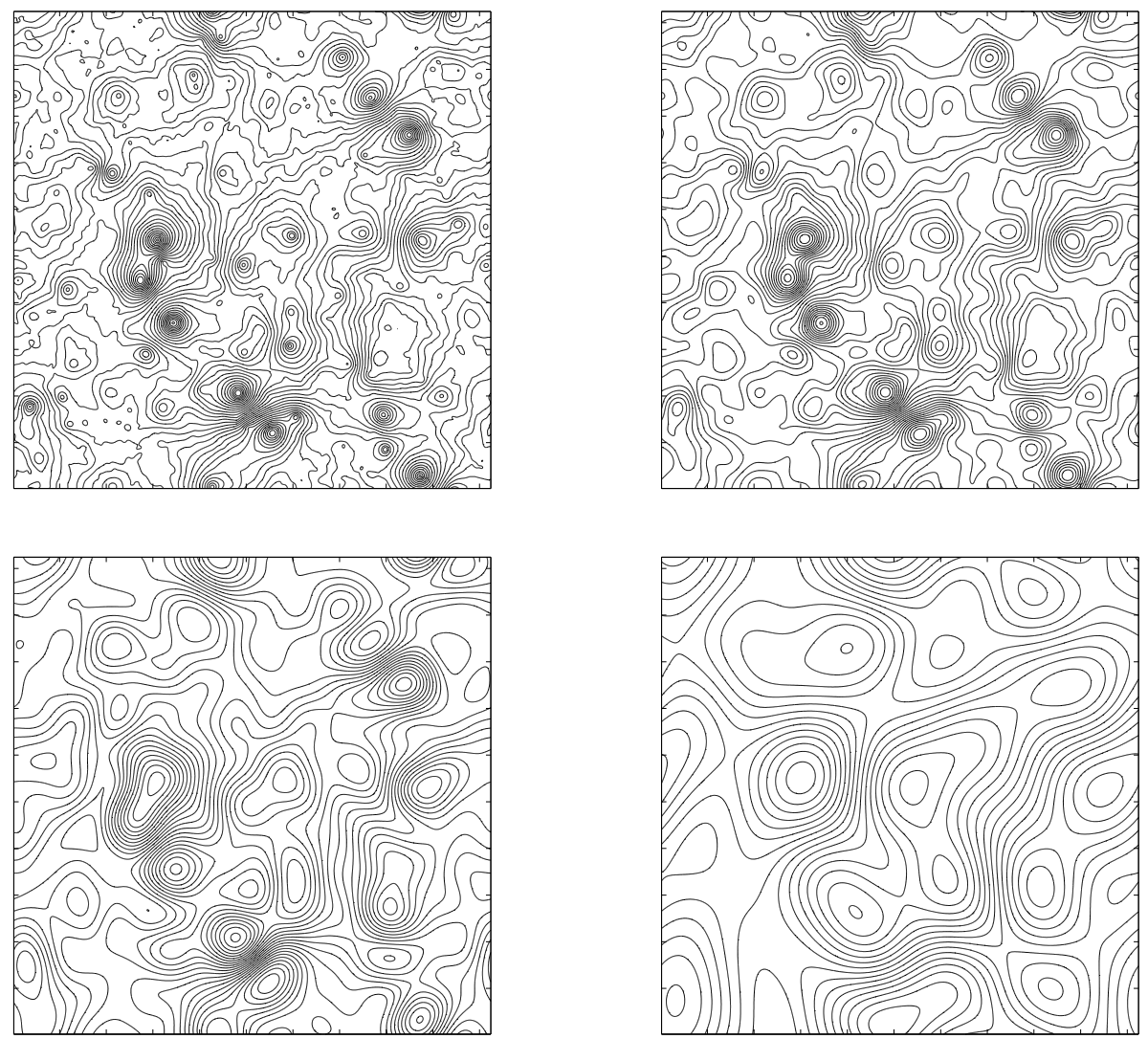

Figure 2: Snapshots of the stream function field for different values of cutoff wavenumber. Top row, $k_{c}=512$ and $k_{c}=32$. Bottom row, $k_{c}=16$ and $k_{c}=8$.

Fig. 3 shows the behavior of the relative dispersion statistic as a function of eddy turnover time for different choices of spectral truncation. Results were obtained by considering nearest neighbor pairs of 40,000 trajectories initialized on a regular grid with initial separation of $2 \pi / 512$ corresponding to $\delta_{0}=1.0$ in the grid-based units shown in all computations and plots. The results are consistent with theory. At large scales, diffusive $D^{2} \sim t$ behavior is observed for all values of the smoothing parameter and the overall 
magnitude of $D^{2}$ is nearly insensitive to the resolution of smaller scale motions. This is true even in the case $k_{c}=16$ where the truncated field only accounts for $60 \%$ of the total flow energy. As such, for times corresponding to mean separations at scales below $2 \pi / k_{c}$ the relative dispersion grows very slowly and, as shown below via doubling time statistics, spatial smoothing corresponds to extending the range of scales over which an exponential separation regime exists. Given the form of the smoothing operator, smooth fields posses infinitely steep spectra for scales $k>k_{c}$ and thus the scale at which the dispersion changes from local to nonlocal (exponential in time) behavior is set directly by the truncation wavenumber $k_{c}$ for all $k_{c}<k_{f}$.

The effect of increasing the role played by large scale structures by spectral truncation is evident in the inset to Fig. 3a which shows Richardson scaling, $D^{2} / t^{3}$, of the computed relative dispersion curves. As indicated by Babiano et al. (1990), experimental observation of Richardson scaling is expected only for particle pairs whose initial separation scale, $D_{0}$, is well below that of the inertial subrange, $D_{i}$. With $D_{0} / D_{i}=0.14$ here, only a very modest regime of Richardson scaling is observed in the full simulation for times $\tau \sim 20-30$. For all other times the growth of the relative dispersion for advection under the full field is slower than $t^{3}$. The dispersion in the smoothed fields, however, asymptotes at large times to that of the full field but initially grows much more slowly due to the complete absence of small scale motions. As such, the rate at which relative dispersion grows at intermediate scales is seen to be significantly greater than the Richardson prediction in the spectrally smoothed fields.
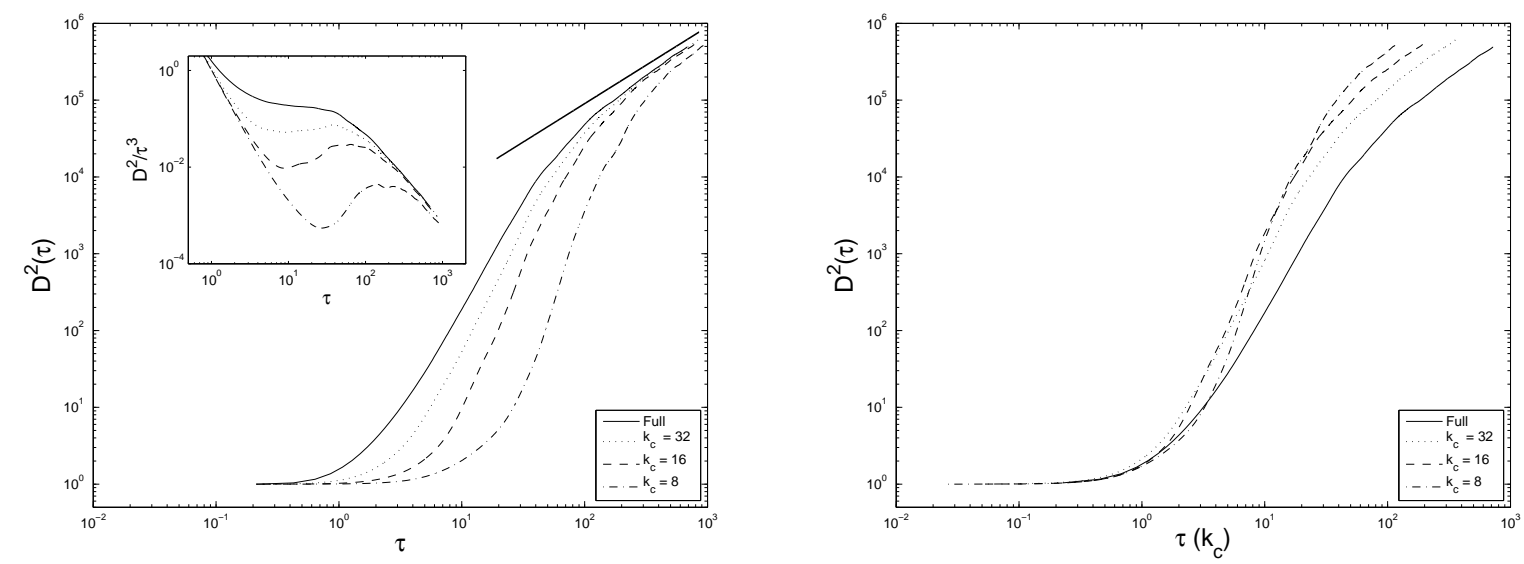

Figure 3: (a) Relative dispersion for full simulation and three values of the cut-off wavenumber. The solid line indicates the diffusive, $D^{2} \sim t$, behavior. Time scaled by eddy-turnover time of the full simulation. Inset shows Richardson scaling, $D^{2} / t^{3}$. (b) Relative dispersion for various smoothing. Time scaled by the root mean square of resolved enstrophy for each value of $k_{c}$.

The behavior of the relative dispersion for short times is further investigated in Fig. 3b. In the ocean modeling context where there is no access to the full solution, it is natural to scale time with available estimates of the eddy-turnover time as computed by the model. Given the cumulative fraction of enstrophy as a function of wavenumber as shown in Fig. 1b, this is easily done in the context of the spectral truncation. As shown in Fig. 3b, rescaling with the resolved enstrophy (equivalent to the resolved rate of strain for the case of homogeneous 2D turbulence) produces very good collapse of the relative dispersion data across smoothing parameters for small times and small separations. At larger scales, however, this local scaling indicates increased dispersion at intermediate and long scaled times for flows lacking small scale structure. In other words, the absence of small scale structure in the flow leads to larger values of relative diffusivity measured with respect to the magnitude of the resolved velocity gradients.

The computed relative dispersion clearly shows the insensitivity of the magnitude of the eddy-diffusivity in the diffusive regime to the presence or absence of small scale motions. Direct calculation of $K(t)=\frac{1}{2} \frac{d D^{2}}{d t}$ from the data confirms that this quantity is identical, within sampling error, for all truncations despite large differences in the resolved kinetic energy $\left(E\left(k_{c}=8\right) \sim .3 E\left(k_{c}=512\right)\right)$. Computation of the Lagrangian kinetic energy and velocity auto-correlation time indicate that the particle auto-correlation times grow with 

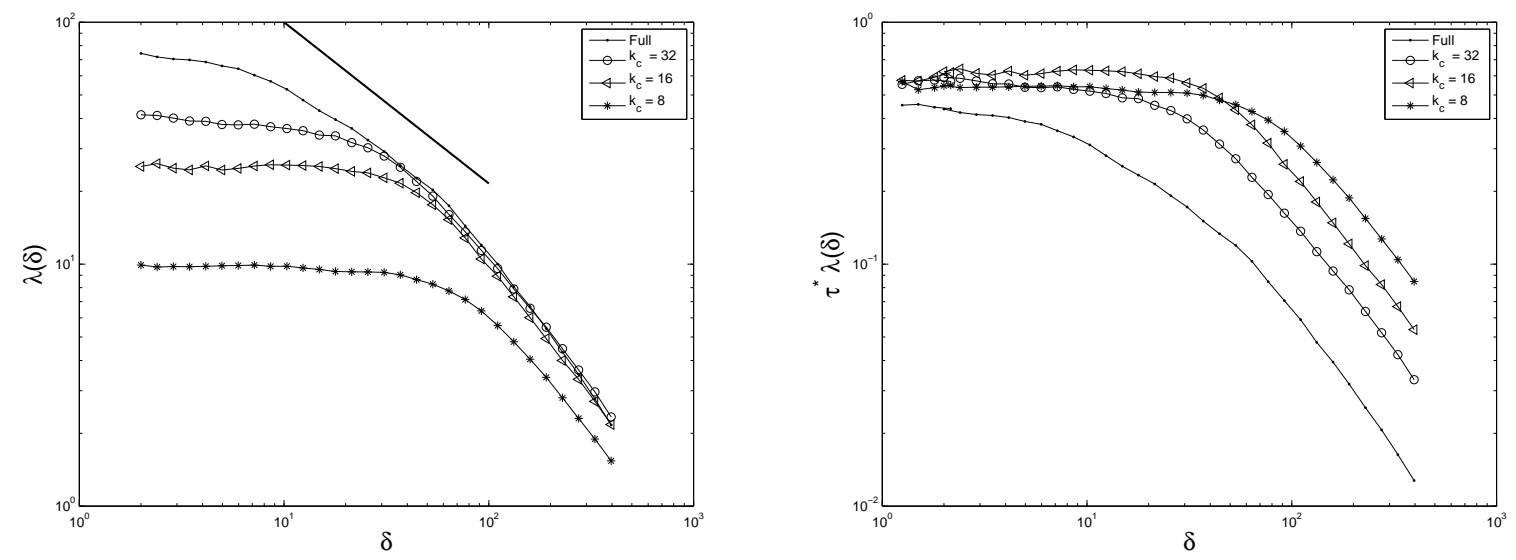

Figure 4: (a) Finite size Lyapunov exponent, $\lambda$ as a function of separation distance $\delta$ for full and smoothed simulations. (b) Same as (a) after scaling $\lambda$ with the resolved enstrophy time scale, $\left(\overline{\omega^{2}}\right)^{1 / 2}$, in each flow.

increasing spatial smoothing in such a way to exactly compensate for the decrease in Lagrangian kinetic energy.

To distinguish the scale dependence of the relative dispersion at small times we calculate the FiniteSize-Lyapunov Exponent (FSLE) originally proposed by Aurell et al. (1997); Artale et al. (1997) and used extensively since then investigations of $2 \mathrm{D}$ turbulence. Instead of averaging over all separation distances at a given time as is done in the calculation of $D^{2}(t)$, the FSLE seeks to compute the average time particle pairs take to separate from a given scale $\delta$ to a scale $\alpha \delta$ where $\alpha>1$ is a parameter. Results for $\alpha=2$ correspond to average doubling times. The FSLE is defined as the inverse time scale

$$
\lambda(\delta)=\frac{\ln (\alpha)}{\langle\tau(\delta)\rangle}
$$

where $\langle\tau(\delta)\rangle$ is the average time over all particle pairs having separated from $\delta$ to $\alpha \delta$. Specifically, we compute the relative dispersion of $2 \times 199 \times 200$ nearest neighbor initial particle pairs and, for each pair, determine the average time to separate a given distance over all occurances of this separation. These per particle pair mean times are then averaged over all pairs to determine $\lambda(\delta)$.

Given the goal of examining the scale dependence of the relative dispersion as a function of spatial velocity resolution, we have chosen the smallest value of $\alpha$ possible in order to observe the scale dependence of dispersion statistics at the finest allowable resolution. As explained in Haza et al. (2008), temporal resolution of trajectory data and statistical considerations place a lower bound on the value of $\alpha$. Specifically, the parameter must not be chosen so small that pair separations occur at times smaller than the time increment of the available data. Given these considerations, in order to resolve the small scale exponential regime with as many distinct separations values as possible we have chosen $\alpha=1.2$. This parameter choice is consistent with Rivera and Ecke (2005) and slightly smaller than typical values $(\alpha=\sqrt{2})$ in Aurell et al. (1997); Lacorata et al. (2001); LaCasce and Ohlmann (2003)). Tests with $\alpha=1.4$ and $\alpha=1.7$ show that the results (at scales resolved by the choice of $\alpha$ ) are insensitive to this parameter.

Results for the 2D turbulence simulations are shown in Fig. 4 where variations in the extent of the observed exponential $\left(\lambda(\delta)=\lambda_{0}=C\right)$ regime with increasing levels of spatial smoothing are clearly seen. Again, at scales larger than $2 \pi / k_{c}$, the FSLE shows very little sensitivity to spatial smoothing and is identical within statistical error for values of the truncation wavenumber greater than 16 . In these cases, average separation times at intermediate separation scales are independent of the presence or absence of smaller scale motions. As seen in Fig. 4a, any indication of Richardson regime, $\lambda \sim \delta^{-2 / 3}$ is lost in the smoothed fields which show an abrupt transition from exponential to diffusive scaling. 
The extent of the exponential regime agrees well with both spectral theory, which predicts constant $\lambda$ for $\delta / 2 \pi<1 / k_{N L}=\max \left(1 / k_{c}, 1 / k_{f}\right)$ and simple ideas of Lagrangian chaos for smooth Eulerian velocity fields. Inspection of Fig. 4a shows, at least for inter-comparison of the three smoothed fields, that the range of the exponential regime scales well with $1 / k_{c}$. As shown in Fig. 4 , the magnitude of the limiting constant value of $\lambda$ scales with the average resolved rate of strain as measured by the local enstrophy in agreement with experimental observations of Rivera and Ecke (2005)
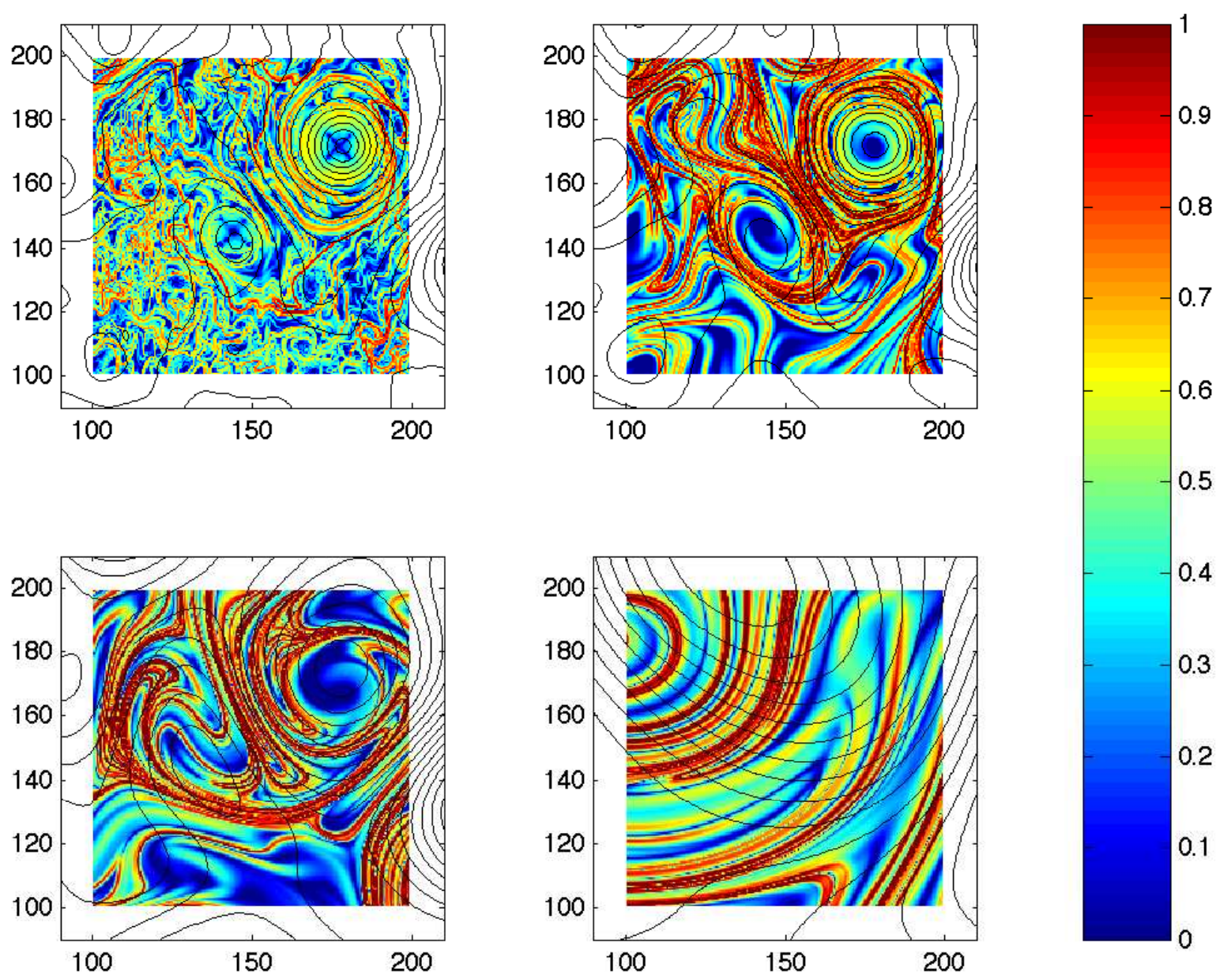

.4
.3
0.1
0

Figure 5: Direct Lyapunov exponent fields showing the effect of spatial smoothing in the Lagrangian frame. The finite time corresponds to approximately 10 eddy-turnover times in each field. Top row, $k_{c}=512$ and $k_{c}=32$. Bottom row, $k_{c}=16$ and $k_{c}=8$. The color scale is identical in each figure.

The behavior of both raw relative dispersion statistics and FSLE distributions under the action of spatial filtering point to the dominant role played by meso-scale structures in determining the rate of particle pair separation at all but the smallest scales. The insensitivity of dispersion measures to the details of the smallscale velocity field has been noted previously (Bracco et al., 2004; Griffa et al., 2004; Haza et al., 2008) and is consistent with the long-held paradigm that large scale structures are responsible for the majority of turbulent mixing.

To investigate the kinematics which allow the seemingly different flow fields shown in Fig. 2 to produce nearly identical large scale dispersion statistics, we compare the geometry of the underlying Lagrangian structures for different spatial smoothings. A number of techniques have been proposed for elucidating the transport geometry in the Lagrangian frame for aperiodic flows (Poje et al., 1999; Haller and Yuan, 
2000; Haller, 2001; Shadden et al., 2005). Here we employ the Direct Lyapunov Exponent (DLE) approach proposed by Haller and used in the context of coastal ocean flows by Lekien et al. (2005).

Given particle trajectories defined by $\mathbf{x}\left(t, \mathbf{x}_{0}\right)$, the direct Lyapunov exponent $\sigma\left(\mathbf{x}_{0}, t_{0}\right)$ is defined as the maximal eigenvalue of the symmetric form of the spatial gradients of $\mathbf{x}$. Define $\lambda_{\max }$ as the largest eigenvalue of

$$
\mathbf{G}=\left[\frac{\partial \mathbf{x}\left(t, \mathbf{x}_{0}\right)}{\partial \mathbf{x}_{0}}\right]^{T}\left[\frac{\partial \mathbf{x}\left(t, \mathbf{x}_{0}\right)}{\partial \mathbf{x}_{0}}\right]
$$

the DLE is then

$$
\sigma\left(\mathbf{x}_{0}, t_{0} ; t\right)=\frac{\ln \left(\lambda_{\max }\right)}{2 *\left(t-t_{0}\right)} .
$$

Figure 5 shows maps of the DLE fields computed for trajectories advected under the full and truncated spectral dynamics. The color scale is identical for all frames. In each, $\mathbf{x}(\mathbf{0}, \mathbf{t})$ is computed on a uniform grid of $200^{2}$ initial conditions and spatial gradients of this flow map are approximated by second order finite differences of trajectory pairs. The DLE is parameterized by the finite time, $t-t_{0}$. Here we have taken this time to correspond to approximately 10 eddy-turnover times as measured by the resolved enstrophy in each truncation. For the particular initialization and subsection of the flow field, the controlling role of a single, large scale vortex dipole pair is made evident as the level of truncation increases up to $k_{c}=16$. Severe truncation at $k_{c}=8$ completely eliminates the dominant meso-scale structure.

\section{Buoyant Coastal Jet}

While the results obtained in the context of idealized 2D flows are useful to develop a better understanding of the resolution dependence of relative dispersion, it is not clear whether these results are specific to this configuration, or carry generality for oceanic flows. In particular, it is desirable to make progress in addressing the following questions: (1) The 2D approach discussed above ensures total homogeneity of the flow field through the boundary conditions and forcing employed. How do relative dispersion characteristics change in more realistic simulations, namely those in which the coherent structures are generated by the instabilities of large-scale features such as coastal and open-ocean jets? (2) Do the main results obtained on the basis of homogeneous fluids change in stratified flows, in which the density is an active tracer in the flow field? (3) While advecting the Lagrangian particles with a reduced number of modes of the same turbulent simulation allows us to identify exactly the effect of small scale turbulent structures on relative dispersion, this is not the traditional practice in ocean modeling. Instead, the typical approach is to increase the spatial resolution of the Eulerian simulation while simultaneously reducing the imposed turbulent viscosity. This, in turn, allows simulation of higher wave number features. How do relative dispersion characteristics vary with this common approach?

To address these questions, we generate a complex flow field using a standard community coastal ocean model. The evolution of an idealized baroclinically-unstable current is simulated using the Regional Ocean Modeling System (ROMS). The main concept of these simulations is to configure the model in a geometrically-simple basin, but in a way to generate complex dynamics involving flow instability. These simulations are instructive to understand the behavior of buoyant coastal currents, such as the Western Adriatic Current along the Italian coast Cushman-Roisin and Korotenko (2007); Book et al. (2007); Haza et al. (2007a); Bignami et al. (2007).

\subsection{Model configuration}

ROMS is a free-surface terrain-following hydrostatic ocean model (Shchepetkin and McWilliams, 2005). ROMS is chosen for three main reasons. First, ROMS is a well-proven model with very good numerics and it is widely used for many coastal applications, so that any results obtained here will have implications for a wider community. Second, ROMS incorporates the convenient feature of implicit viscosity such that the effective Reynolds number at that grid resolution takes the largest value possible while still ensuring stability. The implicit viscosity is built into a third-order, upstream-biased advection operator (Shchepetkin and McWilliams, 1998). In this way, ad-hoc setting of explicit diffusivity for each spatial resolution is 
prevented. Instead, the implicit diffusivity adjusts to the spatial resolution to insure numerical stability. Furthermore, we take advantage of ROMS' feature of being able to deploy on-line synthetic floats so that temporal disctretization errors that can result from off-line deployments are eliminated.

In all simulations, the domain is $580 \mathrm{~km}$ long and $258 \mathrm{~km}$ wide. The southern, eastern and western sides are open, while a no slip condition is applied to the northern closed boundary. The bottom is flat $(H=150 \mathrm{~m})$ and the vertical dimension is discretized by 20 sigma layers. Since the focus is on surface instabilities, the sigma layers are unevenly spaced in the vertical and gathered at the top of the water column. The experiment consists of a set of five numerical simulations which differ only in their horizontal resolution. The resolutions considered are $\Delta x=8 \mathrm{~km}, 4 \mathrm{~km}, 2 \mathrm{~km}, 1 \mathrm{~km}$, and $0.5 \mathrm{~km}$. The baroclinic time-step is held constant at $\Delta t=300 \mathrm{sec}$ except for the $0.5 \mathrm{~km}$ grid where it is decreased to $\Delta t=240$ sec. All simulations are run with the ROMS default generic length scale algorithm (Umlauf et al., 2003) which defines a $k-\varepsilon$ turbulence closure with Canuto-A stability functions (Canuto et al., 2001). Four of the five cases, namely $\Delta x=8 \mathrm{~km}, 4 \mathrm{~km}, 2 \mathrm{~km}$, and $1 \mathrm{~km}$, start from rest. The only forcing is through boundary conditions. The initial salinity and temperature fields vary only with depth according to simple hyperbolic tangent profiles. The surface and bottom initial values are respectively $S^{s r f}=38.1, T^{s r f}=23.25^{\circ} \mathrm{C}$ and $S^{b o t}=38.45, T^{b o t}=13.0^{\circ} \mathrm{C}$. At the eastern side (inflow boundary), in an area $12 \mathrm{~km}$ wide, the tracers are relaxed to fresher and warmer values to simulate the inflow of riverine waters. Near the coast, the salinity and temperature are assigned to the values of $S_{\min }^{s r f}=37.2$ and $T_{m a x}^{s r f}=25.0^{\circ} \mathrm{C}$ in an area $8 \mathrm{~km}$ wide. Moving offshore they gradually match back the initial values. This change follows an exponential behavior with a length scale of $12 \mathrm{~km}$. For brevity, the exact definitions of the initial and boundary profiles are given in the appendix.

If we assume the velocity at the bottom to be zero, the thermal wind equation can be used to calculate the inflow velocity profile from the density field as

$$
u_{E}(y, z)=\int_{-H}^{z} \frac{\partial u}{\partial z} d z=\frac{g}{f \rho_{o}} \int_{-H}^{z} \frac{\partial \rho}{\partial y} d z,
$$

where $\rho_{o}$ is the average density value in the domain, $g$ the gravitational acceleration and $f$ the Coriolis parameter. The barotropic inflow boundary values for the velocity and the sea surface elevation can be then calculated respectively by vertically averaging equation (12) and using geostrophy, i.e.

$$
\begin{aligned}
U_{E}(y) & =\frac{1}{H} \int_{-H}^{0} u_{E}(y, z) d z, \\
\eta_{E}(y) & =\eta_{S}-\frac{f}{g} \int_{0}^{y} U_{E}(y) d y,
\end{aligned}
$$

where $\eta_{S}=0 \mathrm{~m}$ is the sea surface elevation along the southern boundary.

The values $\eta_{E}$ and $U_{E}$ are used at the eastern boundary to specify the incoming characteristic via a Flather condition. The same Flather condition is used at south with $\eta_{S}=0 \mathrm{~m}$ and $U_{S}=0 \mathrm{~m} \mathrm{sec}{ }^{-1}$, while a zero gradient condition is applied on the western boundary for the barotropic velocities. Radiation conditions are applied at the western boundary for the sea surface elevation, and at all open boundaries for the baroclinic velocities. Furthermore, at the western and southern boundaries the tracers are relaxed back to their initial values in two areas that are 48 and $12 \mathrm{~km}$ wide, respectively. At the western boundary, the mesh size is also increased to provide a natural sponge layer and to reduce reflection in the domain.

The simulations with $8 \mathrm{~km}$ and $4 \mathrm{~km}$ grids run for 583 days while the others are terminated at 347 days from rest. The $0.5 \mathrm{~km}$ simulation shares the same setup for the boundary conditions. However, in order to save computational time, it uses as initial conditions the fields calculated by the $2 \mathrm{~km}$ simulation at 208 days and 8 hours. From this point it is integrated in time until the end of the simulation is reached.

\subsection{Results}

In these simulations, the coastal jet is best characterized by its salinity signature, which serves well to visualize the flow field. The eddy-permitting resolution requirement is estimated on the basis of the 

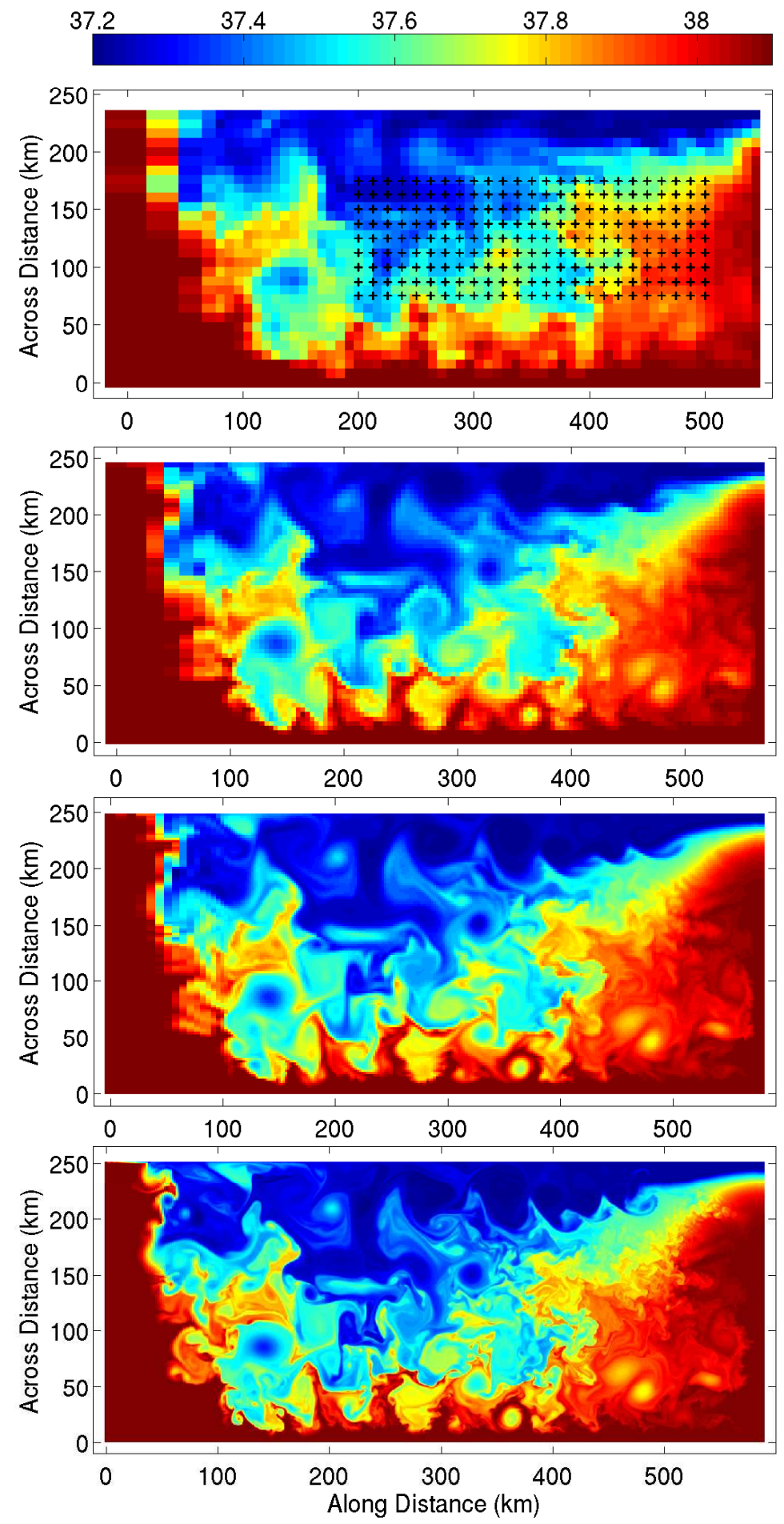

Figure 6: Snapshots of the salinity fields with increasing spatial resolution, (a) $\Delta x=8 \mathrm{~km}$, (b) $\Delta x=4 \mathrm{~km},(\mathrm{c}) \Delta x=2 \mathrm{~km}$ and (d) $\Delta x=0.5 \mathrm{~km}$. The "+" signs in (a) mark the launch locations of the synthetic floats. 
radius of deformation. Using a typical salinity of $S=37.2$ and temperature of $T=25^{\circ} \mathrm{C}$ for the coastal current, and $S=38.1, T=23.25^{\circ}$ for the ambient water, and a linear equation of state with a contraction coefficient of $6.6 \times 10^{-4}$ for salinity, and expansion coefficient of $1.7 \times 10^{-4} C^{-1}$ for temperature, the reduced gravity becomes $g^{\prime}=9.6 \times 10^{-3} \mathrm{~m}^{2} \mathrm{~s}^{-1}$. Also taking $h=25 \mathrm{~m}$ for the buoyant current depth scale, and $f \approx 9.85 \times 10^{-5} \mathrm{~s}^{-1}$ in the domain, the radius of deformation is estimated as $R_{d} \approx 5 \mathrm{~km}$. A resolution of at least $\Delta x=8 \mathrm{~km}$ is required not only to permit meso-scale eddies, but also to capture the width of the buoyant current of $12 \mathrm{~km}$ at the inflow (right) boundary. The snapshots of the salinity field obtained from different spatial resolutions show that the largest eddies are captured adequately regarding their size and phase (Fig. 6). As the spatial resolution is increased, secondary instabilities and more modes of turbulent interactions are captured.

It is of interest to explore the kinetic energy spectrum, as this case is in between the two limiting cases of 2D and 3D turbulence. In purely 2D turbulence, the energy is transferred from small to large scales, while enstrophy is transferred from large to small scales. The injection of energy at a very specific wavenumber, as in section 3 (Fig. 1a) therefore creates a spectrum with a dual range, $E(k) \sim k^{-5 / 3}$ for $k<k_{f}$ and a spectrum with a steeper slope for $k>k_{f}$. The up-scale transfer of energy in 2D requires a large-scale dissipation mechanism, as done in (8). On the other hand, the energy transfer of 3D homogeneous turbulence is down-scale (Kolmogorov, 1941). Regarding relative dispersion, exponential growth occurs when the wave number spectrum is steeper than $k^{-3}$, regardless of the dynamics which set this slope (Bennett, 1984).

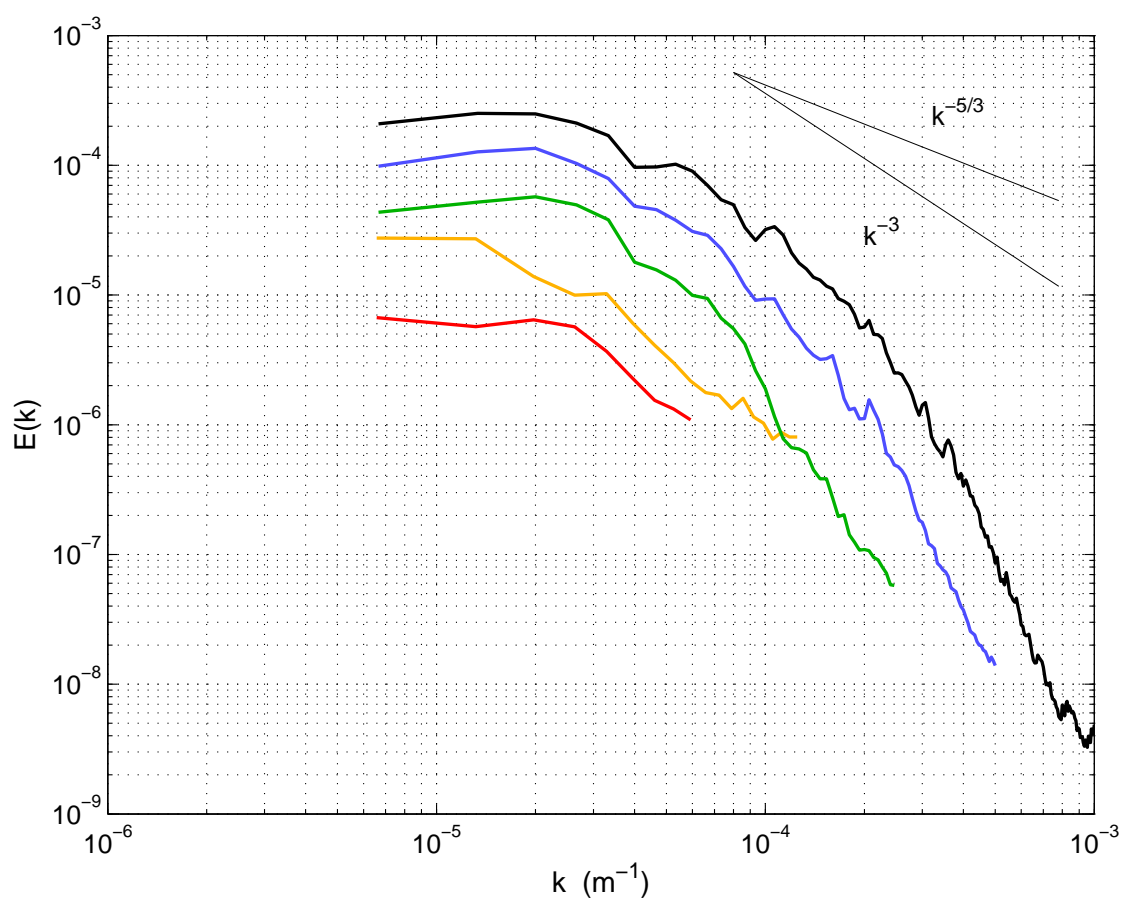

Figure 7: Kinetic energy wave number spectra from the five different resolutions $(8 \mathrm{~km}$ red, $4 \mathrm{~km}$ yellow, $2 \mathrm{~km}$ green, $1 \mathrm{~km}$ blue and $0.5 \mathrm{~km}$ black) of ROMS simulations. Black lines indicate slopes of $k^{-5 / 3}$ and $k^{-3}$.

Kinetic energy wave number spectra for the ROMS simulations are estimated on the basis on 20 sections taken over the area marked in Fig. 6a, which corresponds to the region where the synthetic drifter arrays are launched. The kinetic energy spectra plotted for the five resolutions (Fig. 7) indicate the following. The highest resolution case with $\Delta x=0.5 \mathrm{~km}$ does not show a significant peak at any particular wave number, and two distinct regime as in the forced 2D case (Fig. 1a), but a gradual transition. The regime between $(80 \mathrm{~km})^{-1} \leq k \leq(10 \mathrm{~km})^{-1}$ (consistent with the sizes of large eddies seen in Fig. 6d) has a slope of approximately $k^{-5 / 3}$. In the regime of $k>(9 \mathrm{~km})^{-1}$, the slope becomes steeper. When the spatial resolution is reduced, several changes can be observed. First, and most obviously, the higher wave numbers 
are gradually truncated. In the $8 \mathrm{~km}$ and $4 \mathrm{~km}$ cases, the steeper part of the spectrum is not captured, and only the largest eddies are resolved. Second, there is a significant drop (more than a factor of 10 between $8 \mathrm{~km}$ and $0.5 \mathrm{~km}$ cases) in the overall kinetic energy. In this particular system, baroclinic instabilities act to convert the potential energy stored in the buoyant coastal plume into kinetic energy. Part of this kinetic energy is used for mixing and stored irreversibly as the background potential energy, and part of it is dissipated. In this coastal buoyant plume problem, turbulent instabilities are highly active, and higher resolution cases seem to extract more kinetic energy from the fields, while in coarse resolution cases, implicit subgrid-scale closures provide a direct pathway to mixing and dissipation without explicit turbulence. Ideally, the subgrid-scale closures should be such that the identical results from higher-resolution simulations are reproduced at coarse resolutions. In practice, however, this is usually not the case. So, overall, we conclude that these particular sets of simulations not only have similarities with the 2D flows presented above, but also show significant differences to justify the exploration of the relative dispersion statistics.

A total of 1125 synthetic floats are released in these computations after each simulation has adjusted to its own internal dynamics and developed its own turbulent flow features. The initial locations are chosen to be upstream of the flow field and away from the boundaries Fig. 6a. In an area that is $300 \mathrm{~km}$ long and 100 $\mathrm{km}$ wide, floats are deployed every $12.5 \mathrm{~km}$ and each float is surrounded by four others placed $500 \mathrm{~m}$ apart in each cardinal direction to form a cross. The floats are deployed at the surface and they are kept at their initial depth for the whole duration. The number of floats and their initial positions are identical for all the simulations. The floats trajectories are calculated on-line and their positions are saved with a sampling period of one hour.

The relative dispersion is computed for all cases and plotted as a function of time in Fig. 8a. $D^{2}(t)$ is larger for finer resolutions at any given time. All experiments display an exponential regime followed by a slightly slower than Richardson power-law $\left(D^{2}(t) \sim t^{2.7}\right.$ for the $0.5 \mathrm{~km}$ run). In analogy to the 2-D turbulence results, the exponential regime persists for longer times for decreasing resolution. Unlike the purely 2-D flow, however, there does not appear to be a significant change in the slope of the dispersion curve for different resolutions at intermediate times. Beyond two months, the trend towards a diffusive regime becomes apparent in all but the coarsest resolution.

In all simulations, the presence of an open down-stream boundary through which particles are lost limits the total amount of time over which reliable dispersion statistics can be computed limiting full exploration of the diffusive dispersion regime.

As in the 2D turbulence case, it is desirable to explore whether a resolution-dependent Eulerian quantity sets the maximum exponential relative dispersion at the small scales and the local dispersion time-scale. In inhomogeneous flows, the enstrophy and rate of strain need not be equal. A useful quantity commonly used to characterize the topology of turbulent flows is the Okubo-Weiss criterion (Okubo, 1970; Weiss, 1991)

$$
Q=S^{2}-\omega^{2} .
$$

Here

$$
S^{2}=S_{n}^{2}+S_{s}^{2}, \quad S_{n}=\frac{\partial u}{\partial x}-\frac{\partial v}{\partial y}, \quad S_{s}=\frac{\partial v}{\partial x}+\frac{\partial u}{\partial y},
$$

where $S_{n}$ and $S_{s}$ are the normal and shear components of strain.

Comparison of the Okubo-Weiss parameter computed for the flow fields depicted in Fig. 6 is shown in Fig. 9. The regions dominated by rotation (centers of eddies) are characterized by $Q<0$, while those dominated by strain and deformation (hyperbolic regions in between the eddies) are those where $Q>0$. Regions with $Q<0$ correspond to no or little relative dispersion, while those with $Q>0$ are associated with the exponential divergence of particles. Obviously, $Q$ estimated from frozen fields provides no indications of the time-dependent nature of particle dispersion. Nevertheless, it is clear from Fig. 9 that as the model resolution is increased, hyperbolicity of the model fields increases as well. Since the relative dispersion at small scales is particularly sensitive to the stretching rate, it is sensible to explore whether a measure of regions with $Q>0$ can account for the overall change in the dispersion times with model resolution.

Fig. 8b shows the relative dispersion as a function of scaled time. For each resolution we define an inverse 

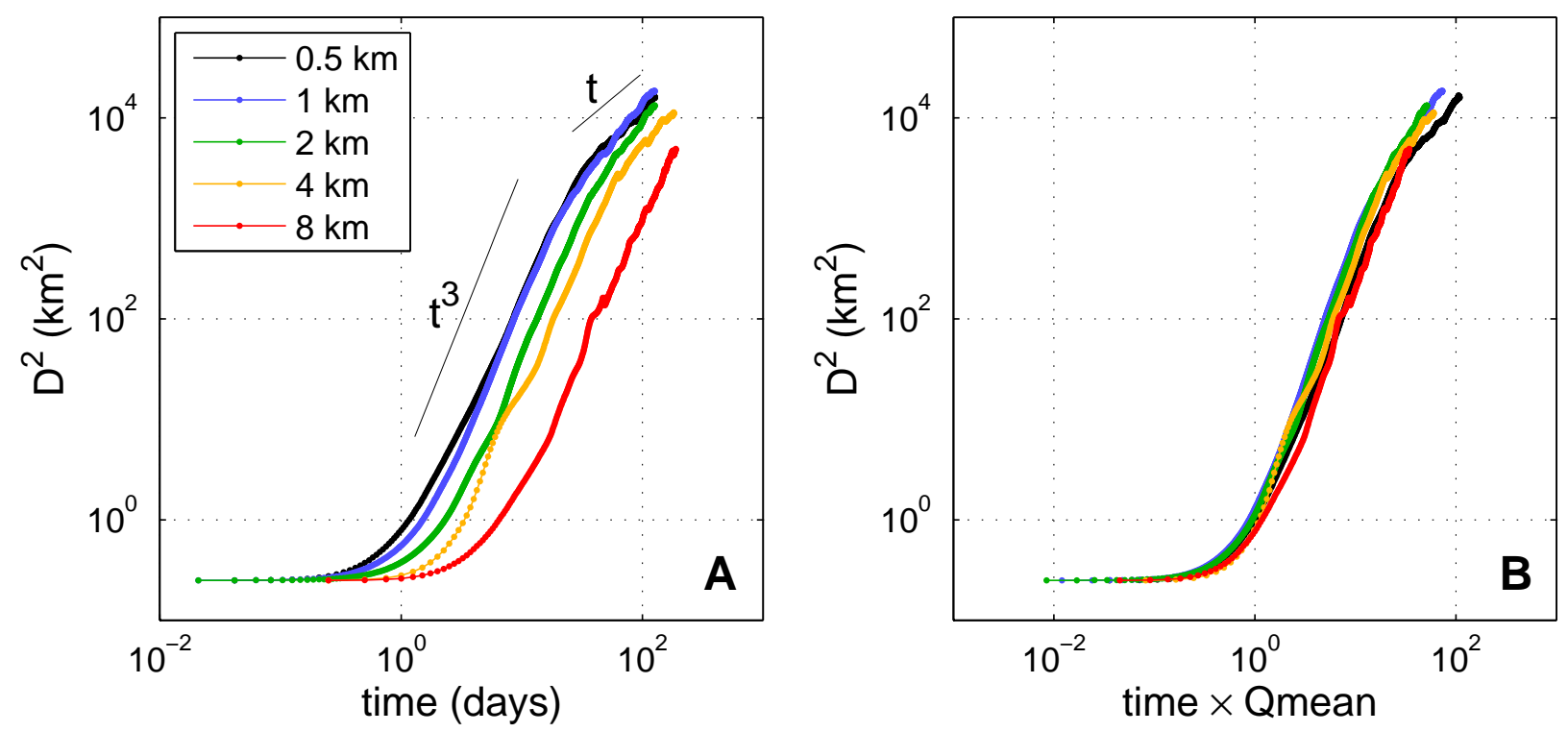

Figure 8: (a) Relative dispersion versus time, $D^{2}(t)$, computed from the five different resolutions of ROMS simulations. (b) Relative dispersion versus rescaled time for the five different resolutions. In each case, time is nondimesionalized by the hyperbolicity time provided by the root of spatially averaged, positive Okubo-Weiss parameter.

time scale, $\bar{Q}$, by averaging over all positive (hyperbolic) values of the Okubo-Weiss partition,

$$
\bar{Q}=A^{-1} \int Q d A, \quad Q>0
$$

As shown in the figure, the relative dispersion in scaled time, $D^{2}(\bar{Q} t)$, shows relatively good collapse across the five spatial resolutions. In agreement with the homogeneous turbulence results, rescaling time with a measure of resolved velocity gradients produces larger relative dispersion in the diffusive regime for coarser resolutions.

The scale dependence of the relative dispersion measured by the FSLE metric is investigated next. The FSLE is based on an averaged time over an ensemble of particle-pairs required to separate from the distances $\delta$ to $\alpha \delta$. There are two non-trivial aspects related to the actual implementation of this definition that deserve some discussion as these details can lead to significant differences in the results. The most common technique in the literature is the first-crossing method, which computes the time elapsed between the first time a particle pair's separation reaches $\delta$ and the following crossing to $\alpha \delta$. Especially in the presence of coherent meso-scale structures, however, there is no guarantee that the separation distance for a given particle pair should increase monotonically with time. For separation distances of the same order as the scales of the energetic structures, the first crossing time between given separations may not be indicative of the average time required for this pair to separate that distance. An alternate approach to pick the last instance a particle pair reaches the distance $\delta$ before reaching the distance $\alpha \times \delta$. This fastest-crossing method gives an averaged time always shorter than the first crossing method and therefore higher corresponding FSLE values. The advantage of the method is that it provides a uniform conditional statement across all pairs and is thus expected to produce more robust statistics. Both methods may yield different FSLE values and, for a finite number of realizations, different power law regimes.

The second issue in the FSLE computation is the particle pair sampling strategy. In light of the limited number of drifters available in oceanic observations. While it is common to rely on chance pairs in order to compute FSLE statistics from limited numbers of observations (LaCasce and Ohlmann, 2003), no such constraints apply to modeling studies. Although Morel and Larcheve (1974) report no statistical difference between chance and original pairs in the relatively homogenous atmospheric context, there is evidence that 
chance pairs introduce a bias in the FSLE computation by preferentially sampling convergence zones in the flow field possibly obscuring the exponential regimes at small scales (e.g., Fig. 4b in Haza et al. (2008)). On the other hand, original pairs can introduce a bias toward an overestimation of the FSLE at larger scales because only pairs in the fastest dispersing regions are preferentially included in the sample given the finite time of the simulation. The difference between the results from original and chance pairs increases when the the flow field is not homogeneous, namely when it includes fast boundary currents next to slow gyres typical in oceanic flows. As such, the use of original pairs at small scales and chance pairs at large scales leads to an optimum sampling to ensure a homogeneous sampling and more reliable statistics of the flow field.

Here we present FSLE $\lambda(\delta)$ results from both original and chance pairs, and also first-crossing and fastestcrossing methods (Fig. 10). Results on the basis of the first-crossing method (Fig. 10a) are qualitatively similar to those obtained from the idealized 2D setting in that they show distinct plateau at small scales with the maximum value of $\lambda$ increasing with spatial resolution. As expected, the fastest crossing method provides a more robust estimate of $\lambda$ at the smallest scales.

The extent of the exponential regime shows dependence on the spatial resolution and extends to larger scales as the resolution decreases. In the highest resolution case, the plateau (seen most clearly Fig. 10a) exists to about $10 \mathrm{~km} \leq \delta \leq 20 \mathrm{~km}$, which is the size of the smallest meso-scale eddies, or 2 to 4 times the radius of deformation. In contrast, for $\Delta x=8 \mathrm{~km} \lambda(\delta)$ is approximately constant for separations less than $60 \mathrm{~km}$.

At larger scales than this, the FSLE shows little sensitivity to the smaller scale motions. At scales $\delta>10 \mathrm{~km}$, original pairs lead to a faster relative dispersion regime, as discussed above (close to Richardson, $\delta^{-2 / 3}$ Richardson) than chance pairs (close to ballistic, $\delta^{-1}$ ). Note that this regime does not entirely coincide with the power-law of $D^{2}(t)$, which is closer to the Richardson regime. When the fastest-crossing method is used (Fig. 10b), the FSLE values are consistently larger (about 5 fold) than those from the first-crossing method.

The FSLE scaled with $\bar{Q}=A^{-1} \int Q d A$ for $Q>0, \lambda / \bar{Q}$, is plotted in Fig. 11 for both first and fastest crossing estimations. In both cases, the curves collapse considerably. This rescaling is more successful with the first-crossing method and reasonable with the fast-crossing method.

\section{The Gulf Stream}

\subsection{Model configuration}

The North Atlantic numerical simulation with the Hybrid Coordinate Ocean Model (HYCOM) uses $1 / 12^{\circ}$ horizontal spacing (Mercator grid merged with bipolar at high latitudes), and 32 vertical layers in sigma2 coordinates (Bleck, 2002; Chassignet et al., 2003; Halliwell, 2004)

The domain extends from $28^{\circ} \mathrm{S}$ to a curvilinear boundary reaching $80^{\circ} \mathrm{N}$. Temperature and salinity fields are relaxed to PHC3.0 climatology in buffer zones near the northern and southern boundaries. The model is forced with ERA40 (1978-2002) monthly climatological forcing. The thermal forcing includes heat flux calculated from bulk formulae for radiation, air temperature, specific humidity, wind speed and model sea surface temperature, and precipitation. In addition, surface salinity is relaxed to climatology. The ERA40 wind stress data were corrected through a correlation with satellite winds, and 6-hourly repeat-year anomalies corresponding to those from 2003 were added. The output analyzed here corresponds to year 5 of the spin-up after initialization from GDEM climatology.

In order to simulate the Gulf Stream realistically, one of the important prerequisites is to correctly represent the separation from the coast. While separation of the Gulf Stream from Cape Hatteras poses a challenge for many coarse-resolution models (Dengg, 1997; Özgökmen et al., 1997) OGCMs with 1/10$1 / 12^{\circ}$ resolution typically capture this behavior quite realistically (Paiva et al., 1999; Smith et al., 2000; Chassignet and Garraffo, 2001) Due to the complexity and high temporal and spatial sampling, the output from such OGCMs take a significant amount of time to study. In investigations conducted on the output of MICOM (Miami Isopycnal Coordinate Model, preceding HYCOM and relatively more extensively studied, e.g. Garraffo et al. (2001b); Veneziani et al. (2005)) the Gulf Stream separation is found to be realistic, and the meso-scale activity in the Gulf Stream region seem to be well represented. Synthetic drifters in regions 
of strong fronts were found to sample velocities that differ from the Eulerian velocities due to convergence effects (Garraffo et al., 2001a). The 3D structure of the confluence region between the Gulf Stream and the Deep Western Current has been studied on the basis of MICOM output by Haza et al. (2007b) and is found to be in good agreement with the observations. One of the main differences between the present HYCOM simulation and those from MICOM is that HYCOM relies more on resolution than parameterization of the oceanic mixed layer flow, because of the six $z$-coordinate layers in the upper few hundred meters. There seems to be no major difference between HYCOM and MICOM in the region of interest, and since MICOM is not supported anymore, we work with HYCOM fields here. These simulations appear to capture well most of the known boundary layer and meso-scale dynamics in this region, while representing the output from fully realistically forced and configured OGCM. Therefore, this model provides an ideal setting for calculating relative dispersion statistics in realistic open ocean flows.

A set of 14,615 particles are launched between Cape Hatteras and the Grand Banks. A set of five particles, $1 \mathrm{~km}$ apart from the central one are released every three grid-points. This corresponds to more than 10,000 original pairs available for computing relative dispersion metrics. A fourth-order Runge-Kutta scheme is used to solve the Lagrangian equations with an integration time-step of $\Delta t=2 h$, and a thirdorder polynomial scheme for spatial interpolation. The total duration of the advection is 4 months, allowing enough particle pairs to cover distances of about $2000 \mathrm{~km}$ (Fig. 12).

There are several differences here with respect to the previous cases, and all concern the computational overhead of this realistic model.

The first difference from the previous sections concerns the representation of the degrees of freedom influencing the two-particle statistics. As discussed above, a coarser-resolution HYCOM simulation of this region would result in a Gulf Stream that overshoots the separation latitude by following the continental shelf until about the Grand Banks, without generating the meso-scale eddies that result from the jet's instabilities between the Cape Hatteras and the Grand Banks. On the other hand, finer resolution experiments are computationally very expensive; the $1 / 12^{\circ}$ discretization consists of $1678 \times 1844$ horizontal points and 32 layers (nearly $10^{8}$ points) with a simulation versus wall clock time ratio of about 100 on 200 CPUs of a Cray XT3 machine. In other words, the present simulation took about 18 days of real time $(86,400$ CPU hours) to complete. A simulation with $1 / 24^{\circ}$ resolution would be approximately 8 times larger; almost $7 \times 10^{5}$ CPU hours and many weeks in real time. Further refinement in resolution is beyond our computational resources at the present time. Thus, being constrained in both directions, towards the coarser and finer grids, we follow the methodology resorted in a previous study (Haza et al., 2008), in which a spatial filtering was used. The spatial filtering of the flow is carried out via a convolution product of the velocity field with a Gaussian function. The spatially-filtered velocity $u^{F}$ at location $i_{0}, j_{0}$ and time step $k$ is:

$$
u^{F}\left(i_{0}, j_{0}, k\right)=\frac{\sum_{i, j} u(i, j, k) e^{\left[\left(i-i_{0}\right)^{2}+\left(j-j_{0}\right)^{2}\right] / \sigma^{2}}}{\sum_{i, j} e^{\left[\left(i-i_{0}\right)^{2}+\left(j-j_{0}\right)^{2}\right] / \sigma^{2}}}
$$

This corresponds to a Gaussian-weighted, spatially-averaged smoothing of the horizontal gradients of the flow with a length scale $\sim 2 \sigma$, while preserving the time dependence. The smoothing parameter values are taken as $\sigma=1.5 ; 3$ and 5 grid points.

Secondly, since the smoothing of the velocity fields can only be done off-line, we rely on off-line advection of particles in all cases. Comparison of 900 on-line and off-line particle releases in the original velocity field did not reveal significant differences in the FSLE curves using the fastest-crossing method and chance pairs. The computational cost of the model limits the total simulation time to a four month time window. As shown below, for the relatively low resolution of the basin-scale simulation as compared to either of the other model flows, this time scale is not long enough to fully investigate the diffusive range of the resulting relative dispersion.

\subsection{Review of relevant observational studies}

Unlike in the previous two test cases, the realism of the HYCOM simulations would allow some comparison of the relative dispersion statistics to the existing observations in this region. The first investigation of relative dispersion in the Gulf Stream region was carried out by Price and Rossby (1982), who found power 
law dependence of relative diffusivity that is consistent with either a Richardson or an exponential regime. A large data set of subsurface drifters released in the Gulf Stream region was analyzed by LaCasce and Bower (2000). In this study, relative diffusivity plots indicated a Richardson regime for separation scales between $10 \mathrm{~km}$ and $100 \mathrm{~km}$ for floats deployed at $600 \mathrm{~m}$ depth. The behavior at larger separation scales was too oscillatory to clearly identify any regimes. One of the primary obstacles in that study appeared to be low number of pairs. Since the floats have not been typically deployed in clusters, their small number resulted in large separation distances on the basis of chance pairs. Subsequently, an area rich with drifter data was targeted by LaCasce and Ohlmann (2003). Analyzing 140 pairs of drifters with a separation distance of less than $1 \mathrm{~km}$ in the Gulf of Mexico, LaCasce and Ohlmann (2003) found two regimes; an exponential regime to about $50 \mathrm{~km}$ or 10 days, and a regime that seems to be in between ballistic and Richardson, depending on whether $D^{2}(t)$ or $\lambda(\delta)$ is used, for longer times and larger separation distances. In the North Atlantic, two other data sets have been generated and analyzed recently. Using a mid-latitude floats in the central part of the North Atlantic, Ollitrault et al. (2005) found a Richardson regime for separation distances between 40 and $300 \mathrm{~km}$. A data set of 55 surface drifters launched in pairs and trios in the Gulf Stream region and tracked with hourly sampling was investigated by Lumpkin and Ellipot (2009), who conclude that these drifters exhibit Richardson's law dispersion from 1-3 km to $300-500 \mathrm{~km}$. This study also concludes that drifters do not exhibit exponential separation at scales larger than 1-3 km.

\subsection{Results}

Before we present the relative dispersion results, we first investigate the effect of the spatial filtering on the flow field using Okubo-Weiss maps in order to see whether there is any significant difference with respect changing model resolution. Fig. 13 appears to indicate that the result are qualitatively similar to those seen in Fig. 9.

While the existence of an initial exponential regime is generally hard to quantify from $D^{2}(t)$ curves, we find in all cases a $D^{2} \sim t^{3}$ power-law corresponding to the Richardson regime for $t \geq 3$ days (Fig. 14). As also shown in Haza et al. (2008), the spatial filtering of the flow field slows down the dispersion speed at a given time and tends to prolong the initial transition regime. This is generally consistent with the trends in $D^{2}(t)$ seen in other sections, namely when synthetic drifters are advected with a truncated modes in the 2D turbulence model (Fig. 3a) and when the resolution is changed in ROMS (Fig. 8).

As in ROMS experiments, both first- and fastest-crossing methods are used to compute the FSLE. The full field FSLE (Fig. 15) displays an exponential regime for the scales $1 \mathrm{~km} \leq \delta \leq 100 \mathrm{~km}$, that is for the scales smaller than two to three times the radius of deformation in this region, $35 \mathrm{~km} \leq R_{d} \leq 50 \mathrm{~km}$. Beyond that critical scale, the curve describes a power-law regime.

It is found that the FSLE plateau is reduced with increasing filtering, while the large scale dispersion remains the same, as in previous sections. The first-crossing method in Fig. 15a yields a power law very close to the Richardson regime $\left(\lambda \sim \delta^{-2 / 3}\right)$, while the fastest-crossing method (Fig. 15b) displays a power-law closer to the ballistic regime $\left(\lambda \sim \delta^{-1}\right)$. The difference in power-law between both methods is in part due to the more pronounced tendency of particles to wiggle in the small scales than in the large scales, thereby relatively slowing further the dispersion time of particle-pairs at the small scales by reducing the plateau value, and lifting the slope of the larger scale FSLE.

Noting that chance pairs have better properties for computing large scale statistics, the FSLE curves for all experiments are estimated also on the basis of chance pairs. The results show that the slope of $\lambda(\delta)$ becomes a bit steeper for $\delta>100 \mathrm{~km}$ in the case of the first-crossing method (Fig. 15a) while there is no significant change with the fastest-crossing method (Fig. 15b). This is the same trend as observed in the ROMS computations. With the chance pairs, filtering reduces the FSLE plateau, while the behavior at large scales remains unchanged. As expected, we conclude that the fastest-crossing method is less sensitive to the differences between original and chance pairs than the first-crossing method.

Following the arguments outlined in Section $4, \lambda$ is rescaled by $\bar{Q}$ and the results are displayed in Fig. 16 . The collapse of the FSLE plateau occurs effectively with the fastest-crossing method (Fig. 16b), while it is

less effective with the first-crossing method (Fig. 16a). Since the exponential regime is a signature of the hyperbolicity of the flow field, one can assume that the fastest crossing method in this particular HYCOM 
simulation is more suited to capture the relative dispersion from the hyperbolic regions of the eddy-field. These results show a trend somewhat opposite to the ROMS experiments. It is either due to a difference in the nature of the circulation, or to the simple fact that altering the stretching rate of the velocity field by applying low-pass filters is not a dynamical equivalent of changing the numerical horizontal resolution.

How do HYCOM results compare with the existing observations? The only data set with which we can make direct comparisons is that of Lumpkin and Ellipot (2009). The FSLE curves shown in Fig. 15 are in excellent quantitative agreement (both the slope and magnitude of the $\lambda(\delta)$ ) with their result for $\delta>200 \mathrm{~km}$. For $\delta<200 \mathrm{~km}$, the model encounters the exponential regime in which the magnitude of the maximum FSLE is determined by the strain set in the hyperbolic regions between the meso-scale eddies, whereas observations indicate an extension of the Richardson regime down to $3 \mathrm{~km}$ so that $\lambda(\delta=3 \mathrm{~km}) \approx 10$ days $^{-1}$, or some 20 times faster than modeled here. The most obvious reasons for this discrepancy are several missing ingredients in HYCOM with respect to oceanic conditions. As the authors state, this experiment was conducted under extreme weather conditions, where winds were probably strong enough for flow conditions to deviate from an approximate geostrophic balance and lead to submeso-scale instabilities. These instabilities may have extended the Richardson's regime to much smaller scales than the meso-scale eddy scale. While the level of the exponential regime does not compare well to that measured by Lumpkin and Ellipot (2009), we note that the $e$-folding time of roughly 2 days is in agreement with that inferred by LaCasce and Ohlmann (2003) in the Gulf of Mexico with the SCULP data. High winds may have also caused wave-driven Stokes drift, as well as flow divergence and other small-scale processes that are certainly missing in this model. Overall, the identification of an exponential regime of the relative dispersion in oceanic observations remains elusive, either due to drifter launch and sampling limitations, or simply the challenge of developing a basic understanding of the oceanic multi-scale turbulent fields subject to complex forcing conditions, especially due to the lack of Eulerian fields simultaneous with drifter data. These differences between the models and observations at the submeso-scales should in principle serve as excellent guidelines for model development. Nevertheless, the existing hierarchy of the numerical simulations reveal a fairly consistent picture; the relative dispersion arising from the flow field is always divided into two regimes determined by the large coherent turbulent structures. The relative dispersion at scales larger than the eddy scale is not sensitive to small-scale processes while relative dispersion at scales smaller than the eddy scale is characterized by an exponential regime, where the value of the fastest dispersion is given by the highest strain rate resolved in the model. In Fig. 17, the values of $\lambda_{\max }$ in the exponential regimes of the ROMS and HYCOM simulations are plotted as a function of $\bar{Q}$. The scatter points collapse well along the lines depending on whether firstor fastest-crossing method is used.

\section{Summary and Conclusions}

The time and scale dependence of the relative dispersion of particle pairs is a fundamental Lagrangian measure which ocean models are increasingly asked to predict. Our main objective has been to quantify the sensitivity of this particle-based statistic to changes in the spatial resolution of the underlying Eulerian model data. To accomplish this we have examined the output from three standard models of increasing complexity and realism. First, we consider a highly idealized, statistically steady, homogeneous and isotropic turbulence in 2-D. For this flow, resolution effects can be readily studied via spectral truncations of the full dynamics. Next, we consider a process model of a baroclinic wall jet simulated with ROMS. Intrinsic sub-grid scale closures allow examination of distinctly different spatial resolutions at fixed values of the input forcing. Finally, we examine the output of HYCOM in the North Atlantic basin. In this case, variations in model spatial resolution are achieved by post-processing the output velocity fields with spatial filters of increasing scale.

In terms of the resulting dispersion statistics of particle pairs and their sensitivity to model resolution, there are a number of results which are universal across the three disparate flows. As expected, short time and small scale pair separations are most sensitive to the details of the advecting flow, and thus most dependent on the spatial resolution of the model. In all flows, the elimination of small scale features implies an increase in the exponential dispersion regime where the logarithmic rate of separation is independent of the separation distance. This is clearly seen in calculations of Lyapunov exponents. In each model, the 
limiting small-scale value of the finite-size Lyapunov exponent was found to be dependent on the resolution of spatial velocity gradients in hyperbolic regions of the flow field. Simple rescaling of the dispersion times with the average value of the resolved enstrophy (in the case of homogeneous turbulence), or the average positive value of the Okubo-Weiss parameter (for the inhomogeneous flows) produces very good collapse of the limiting stretching exponent. Similar resolution dependent rescaling of time indicates that the shape of the relative dispersion curves are self similar during the initial, scale independent phase.

At long time and large space scales all three flows showed the expected insensitivity of the relative dispersion to the resolution of small scale motions. This was most apparent in the 2-D turbulence case where the simplicity of the model and the closed flow domain allow for almost arbitrarily long simulations and full resolution of the diffusive limit. In the diffusive regime, the eddy-diffusivity provided by the relative dispersion is essentially a single point statistic which depends only on the Lagrangian kinetic energy and velocity auto-correlation time,

$$
K=\frac{1}{2} \frac{d D^{2}}{d t} \approx 2 \int_{t_{0}}^{t}\left\langle\mathbf{v}(\mathbf{a}, t) \cdot \mathbf{v}\left(\mathbf{a}, t^{\prime}\right)\right\rangle_{a} d t^{\prime}=4 E_{l a g} T_{l a g} .
$$

Detailed calculations in the 2-D turbulence case where the limiting relative diffusivities are identical for all spectral truncations considered shows that the Lagrangian time-scale grows with decreasing resolution to exactly compensate the decrease in kinetic energy. Similar behavior of the particle correlation time appears to exist in the baroclinic ROMS simulation where small changes in Eulerian model resolution produce large changes in the kinetic energy but have very little effect on relative dispersion at large times.

Universal conclusions are less clear at intermediate time and space scales where the behavior of the relative dispersion is sensitive to the detailed dynamics of meso-scale flow features. Indeed, there are several indications that the effects of spatial smoothing at the grid scale effect the relative dispersion at significantly larger scales. In the 2-D turbulence, elimination of the structures at the small scale end of the inertial range completely eliminates the Richardson regime observed in the full dynamics. Indeed, the removal of all but the largest scales of motion leaves the diffusive regime unchanged while extending the exponential regime which implies that suppressing small scale motions leads to faster growth of the relative dispersion at intermediate times. This behavior is less noticeable in the ROMS simulation, where the power-law scaling of $K(t)$ was found to be somewhat less than the Richardson prediction but approximately invariant across resolutions.

The simple rescaling of time which unifies the results at small scales does not lead to a collapse of the data at intermediate and long times. For fixed values of rescaled time in the non-exponential regime, each model indicated an increase in the relative dispersion with decreasing spatial resolution.

On the whole, these results are encouraging for ocean models - the large scale, long time diffusive behavior is found to be extremely insensitive to resolution while modifications to the short time regime can be properly accounted for by considering the spatial resolution of Eulerian measures of velocity gradients. There are, however, some caveats. (1) In each flow, the resolution/spatial smoothing operators studied have been constructed to leave the time-dependence of the resolved flow as close to that of the reference simulation as possible (note the similarity of the phase information across resolutions in ROMS in (Fig. 6). This may not be the case for model data comparisons in the ocean context or for model-model comparisons where spatial truncation significantly alters the time dependence of the large-scale motion. (2) At intermediate timescales, the overall behavior of the relative dispersion is resolution dependent with this dependence felt even at separation distances much larger than the grid-scale. The increase in the extent of the exponential separation regime and the modification of the growth rate impact the shape and strength of the resulting 'Lagrangian Coherent Structure' boundaries computed via dynamical systems techniques. (3) The insensitivity of the resulting eddy-diffusivity to resolution implies that care should be taken when estimating Lagrangian timescales from coarsely resolved models since these will necessarily be overestimated when the total Lagrangian kinetic energy is underestimated.

\section{Acknowledgments}


We are grateful to ONR via grants N00014-05-1-0094 and N00014-05-1-0095 (Haza, Özgökmen), N0001400-0019 (Poje), and to NSF via ATM-200102 (Poje). Z. Garraffo's participation was funded through University of Miami's Center for Computational Science. We thank Rick Lumpkin for several thought-provoking discussions and exchanges of ideas.

\section{A. Initial conditions in ROMS}

The initial salinity and temperature profiles are:

$$
\begin{aligned}
& S(x, y, z, t=0)=a_{1}^{i n i}+a_{2}^{i n i} \tanh \left(\frac{z+h_{c}^{i n i}}{h_{d}}\right), \\
& T(x, y, z, t=0)=b_{1}^{i n i}+b_{2}^{i n i} \tanh \left(\frac{z+h_{c}^{T}}{h_{d}}\right),
\end{aligned}
$$

where $h_{c}^{i n i}=25 \mathrm{~m}, h_{c}^{T}=22 \mathrm{~m}, h_{d}=5 \mathrm{~m}$,

$$
\begin{aligned}
& a_{1}^{i n i}=\frac{S^{s r f}+S^{b o t}}{2}, \\
& a_{2}^{i n i}=a_{1}^{i n i}-S^{b o t} \\
& b_{1}^{i n i}=\frac{T^{s r f}+T^{b o t}}{2}, \\
& b_{2}^{i n i}=b_{1}^{i n i}-T^{b o t},
\end{aligned}
$$

and $S^{s r f}=38.1, S^{b o t}=38.45, T^{s r f}=23.25^{\circ} \mathrm{C}$ and $T^{b o t}=13.0^{\circ} \mathrm{C}$.

At the eastern (inflow) boundary, if $Y_{\max }=250 \mathrm{~km}, Y_{0}=8 \mathrm{~km}$ and $L_{d}=12 \mathrm{~km}$, the tracers are relaxed to the following profiles:

$$
\begin{aligned}
& S\left(x=x_{E}, y, z, t\right)=a_{1}(y)+a_{2}(y) \tanh \left(\frac{z+h_{c}(y)}{h_{d}}\right), \\
& T\left(x=x_{E}, y, z, t\right)=b_{1}(y)+b_{2}(y) \tanh \left(\frac{z+h_{c}^{T}}{h_{d}}\right),
\end{aligned}
$$

where if $y \leq Y_{\max }-Y_{0}$

$$
\begin{aligned}
& a_{1}(y)=a_{1}^{i n i}-\frac{\Delta S}{2} \exp \left(\frac{y-Y_{\max }+Y_{0}}{L_{d}}\right), \\
& a_{2}(y)=a_{1}(y)-S^{b o t}, \\
& b_{1}(y)=b_{1}^{i n i}+\frac{\Delta T}{2} \exp \left(\frac{y-Y_{\max }+Y_{0}}{L_{d}}\right), \\
& b_{2}(y)=b_{1}(y)-T^{b o t}, \\
& h_{c}(y)=h_{c}^{i n i}-\Delta h \exp \left(\frac{y-Y_{\max }+Y_{0}}{L_{d}}\right),
\end{aligned}
$$

otherwise

$$
\begin{aligned}
& a_{1}(y)=a_{1}^{i n i}-\frac{\Delta S}{2}, \\
& a_{2}(y)=a_{1}(y)-S^{b o t}, \\
& b_{1}(y)=b_{1}^{i n i}+\frac{\Delta T}{2}, \\
& c_{2}(y)=b_{1}(y)-T^{b o t}, \\
& h_{c}(y)=h_{c}^{i n i}-\Delta h,
\end{aligned}
$$

and $\Delta S=0.9, \Delta T=1.75^{\circ} \mathrm{C}$ and $\Delta h=13 \mathrm{~m}$. 


\section{References}

Abraham, E. R. and M. M. Bowen. Chaotic stirring by a mesoscale surface-ocean flow. Chaos, 12:373-381, Jun 2002.

Artale, V., G. Boffetta, A. Celani, M. Cencini, and A. Vulpiani. Dispersion of passive tracers in closed basins: Beyond the diffusion coefficient. Phys. Fluids, 9:3162-3171, NOV 1997.

Aurell, E., G. Boffetta, A. Crisanti, G. Paladin, and A. Vulpiani. Predictability in the large: An extension of the concept of Lyapunov exponent. J. Phys. A, 30:1-26, JAN 71997.

Babiano, A., C. Basdevant, P. Leroy, and R. Sadourny. Relative dispersion in 2-dimensional turbulence. J. Fluid Mech., 214: 535-557, May 1990. ISSN 0022-1120.

Batchelor, G. K. Diffusion in a field of homogeneous turbulence 2. the relative motion of particles. Proc. Cambridge Phil. Soc., 48(2):345-362, 1952.

Bennett, A. F. Relative dispersion - local and nonlocal dynamics. J. Atmos. Sci., 41(11):1881-1886, 1984. ISSN $0022-4928$.

Beron-Vera, F. J., M. J. Olascoaga, and G. J. Goni. Oceanic mesoscale eddies as revealed by Lagrangian coherent structures. Geophys. Res. Lett., 35(12):7, Jun 2008.

Bignami, F., R. Sciarra, S. Carniel, and R. Santoleri. Variability of Adriatic Sea coastal turbid water from SeaWiFS imagery. J. Geophys. Res., 112:C03S10, doi:10.1029/2006JC003518, 2007.

Bleck, R. An oceanic general circulation model framed in hybrid isopycnic cartesian coordinates. Ocean Modelling, 4:55-88, 2002.

Book, J., R. Signell, and H. Perkins. Measurements of storm and nonstorm circulation in the northern Adriatic: October 2002 through April 2003. J. Geophys. Res., 112:doi:10.1029/2006JC003556, 2007.

Bracco, A., J. von Hardenberg, A. Provenzale, J. B. Weiss, and J. C. McWilliams. Dispersion and mixing in quasigeostrophic turbulence. Phys. Rev. Lett., 92(8):084501, Feb 2004. ISSN 0031-9007.

Canuto, V. M., A. M. Howard, Y. Cheng, and M. S. Dubovikov. Ocean turbulence. part i: One-point closure model momentum and heat vertical diffusivities. J. Phys. Oceanogr., 31:1413-1426, 2001.

Castilla, R., J. M. Redondo, P. J. Gamez-Montero, and A. Babiano. Particle dispersion processes in two-dimensional turbulence: a comparison with 2-d kinematic simulation. Nonlinear Proc. Geophys., 14(2):139-151, 2007. ISSN 1023-5809.

Chassignet, E. and Z. Garraffo. Viscosity parameterization and the gulf stream separation. In "From Stirring to Mixing in a Stratified Ocean". Proceedings 'Aha Huliko'a Hawaiian Winter Workshop., P. Muller and D. Henderson, Eds.:37-41, 2001.

Chassignet, E., L. Smith, G. Halliwell, and R. Bleck. North Atlantic simulation with the HYbrid Coordinate Ocean Model (HYCOM): Impact of the vertical coordinate choice, reference density, and thermobaricity. J. Phys. Oceanogr., 33:2504-2526, 2003.

Coulliette, C., F. Lekien, J. D. Paduan, G. Haller, and J. E. Marsden. Optimal pollution mitigation in Monterey Bay based on coastal radar data and nonlinear dynamics. Environ. Sci. Technol., 41(18):6562-6572, Sep 2007. ISSN 0013-936X.

Cushman-Roisin, B. and K. Korotenko. Mesoscale-resolving simulations of summer and winter bora events in the adriatic sea. J. Geophys. Res., 112:doi:10.1029/2006JC003516, 2007.

Dengg, J. The problem of Gulf Stream separation: a barotropic approach. J. Phys. Oceanogr., 23:2182-2200, 1997.

d'Ovidio, F., V. Fernandez, E. Hernandez-Garcia, and C. Lopez. Mixing structures in the Mediterranean Sea from finite-size Lyapunov exponents. Geophys. Res. Lett., 31, SEP 22004.

Ehlmaidi, D., A. Provenzale, and A. Babiano. Elementary topology of two-dimensional turbulence from a Lagrangian viewpoint and single-particle dispersion. J. Fluid Mech., 257:533-558, 1993.

Garraffo, Z., A. Griffa, A. Mariano, and E. Chassignet. Lagrangian data in a high resolution numerical simulation of the north atlantic. ii: On the pseudo-eulerian averaging of lagrangian data. J. Mar. Sys., 29:177-200, 2001a.

Garraffo, Z., A. Mariano, A. Griffa, C. Veneziani, and E. Chassignet. Lagrangian data in a high resolution numerical simulation of the north atlantic. i: Comparison with in-situ drifter data. J. Mar. Sys., 29:157-176, 2001b.

Garrett, C. Turbulent dispersion in the ocean. Prog. Ocean., 70:113-125, 2006.

Griffa, A., L. Piterbarg, and T. Özgökmen. Predictability of Lagrangian particle trajectories: Effects of smoothing of the underlying Eulerian flow. J. Mar. Res., 62(1):1-35, Jan 2004. ISSN 0022-2402.

Haller, G. Lagrangian structures and the rate of strain in a partition of two-dimensional turbulence. Phys. Fluids, 13(11): 3365-3385, Nov 2001. ISSN 1070-6631.

Haller, G. and G. Yuan. Lagrangian coherent structures and mixing in two-dimensional turbulence. Phys. D, 147:352-370, Dec 152000.

Halliwell, G. Evaluation of vertical coordinate and vertical mixing algorithms in the hybrid coordinate ocean model (hycom). Ocean Modelling, 7:285-322, 2004.

Haza, A., A. Griffa, P. Martin, A. Molcard, T. Özgökmen, A. Poje, R. Barbanti, J. Book, P. Poulain, M. Rixen, and P. Zanasca. Model-based directed drifter launches in the adriatic sea: results from the DART experiment. Geophys. Res. Letters, 34:doi:10. 1029/2007GL029634, 2007a.

Haza, A. C., A. J. Mariano, T. M. Chin, and D. B. Olson. The mean flow and variability of the gulf stream-slopewater system from micom. Ocean Modelling, 17/3:239-276, 2007b.

Haza, A. C., A. C. Poje, T. M. Özgökmen, and P. Martin. Relative dispersion from a high-resolution coastal model of the Adriatic Sea. Ocean Modelling, 22(1-2):48-65, 2008.

Iudicone, D., G. Lacorata, V. Rupolo, R. Santoleri, and A. Vulpiani. Sensitivity of numerical tracer trajectories to uncertainties in ogcm velocity fields. Ocean Modelling, 4(3-4):313 - 325, 2002. ISSN 1463-5003.

Kolmogorov, A. The local structure of turbulence in incompressible viscous fluids for very large Reynolds number. Dokl. Akad. Nauk SSR, 30:9-13, 1941.

LaCasce, J. and A. Bower. Relative dispersion in the subsurface North Atlantic. J. Mar. Res., 58:863-894, 2000. 
LaCasce, J. H. Statistics from Lagrangian observations. Prog. Ocean., 77/1:1-29, 2008.

LaCasce, J. H. and C. Ohlmann. Relative dispersion at the surface of the Gulf of Mexico. J. Mar. Res., 61(3):285-312, May 2003. ISSN 0022-2402.

Lacorata, G., E. Aurell, and A. Vulpiani. Drifter dispersion in the Adriatic Sea: Lagrangian data and chaotic model. Annales Geophysicae, 19(1):121-129, Jan 2001. ISSN 0992-7689.

Lekien, F., C. Coulliette, A. Mariano, E. Ryan, L. Shay, G. Haller, and J. Marsden. Pollution release tied to invariant manifolds: A case study for the coast of florida. Physica D: Nonlinear Phenomena, 210(1-2):1-20, Oct 2005.

Lipphardt, B. L., D. Small, A. D. Kirwan, S. Wiggins, K. Ide, C. E. Grosch, and J. D. Paduan. Synoptic Lagrangian maps: Application to surface transport in Monterey Bay. J. Mar. Res., 64(2):221-247, Mar 2006. ISSN 0022-2402.

Lumpkin, R. and S. Ellipot. Surface drifter pair spreading in the north atlantic. J. Fluid Mech., page submitted, 2009.

Mancho, A., E. Hernández-García, D. Small, S. Wiggins, and V. Fernández. Lagrangian Transport through an Ocean Front in the Northwestern Mediterranean Sea. J. Phys. Oceanogr., 38(6):1222, Jan 2008.

Morel, P. and M. Larcheve. Relative dispersion of constant-level balloons in 200-MB general circulation. Journal of the Atmospheric Sciences, 31(8):2189-2196, 1974.

Okubo, A. Horizontal dispersion of floatable particles in vicinity of velocity singularities such as convergences. Deep-Sea Res., 17(3):445-\&, 1970

Olascoaga, M. J., I. I. Rypina, M. G. Brown, F. J. Beron-Vera, H. Koçak, L. E. Brand, G. R. Halliwell, and L. K. Shay. Persistent transport barrier on the West Florida Shelf. Geophys. Res. Lett., 33:22603-+, Nov. 2006.

Ollitrault, M., C. Gabillet, and A. C. DeVerdiere. Open ocean regimes of relative dispersion. J. Fluid Mech., 533:381-407, 2005.

Özgökmen, T., E. Chassignet, and A. Paiva. Impact of wind forcing, bottom topography, and inertia on mid-latitude jet separation in a quasi-geostrophic model. J. Phys. Oceanogr., 27:2460-2476, 1997.

Paiva, A., J. Hargrove, E. Chassignet, and R.Bleck. Turbulent behavior of a fine mesh (1/12 degree) numerical simulation of the North Atlantic. J. Mar. Sys., 21:307-320, 1999.

Poje, A., G. Haller, and I. Mezic̀. The geometry and statistics of mixing in aperiodic flows. Phys. Fluids, 11:2963-2968, 1999.

Price, J. and T. Rossby. Observation of a planetary wave during the local dynamics experiment. J. Mar. Res., 40:543-558, 1982.

Richardson, L. F. Atmospheric diffusion shown on a distance-neighbour graph. Proc. Royal Soc. London: Series A, 110(756): 709-737, Apr 1926. ISSN 0950-1207.

Rivera, M. K. and R. E. Ecke. Pair dispersion and doubling time statistics in two-dimensional turbulence. Phys. Rev. Letters, 95(19):194503, Nov 2005. ISSN 0031-9007.

Sawford, B. Turbulent relative dispersion. Ann. Rev. of Fluid Mech., 33:289-317, 2001. ISSN 0066-4189.

Shadden, S., F. Lekien, and J. Marsden. Definition and properties of Lagrangian coherent structures from finite-time Lyapunov exponents in two-dimensional aperiodic flows. Phys. D, 212(3-4):271-304, Dec 2005.

Shchepetkin, A. F. and J. C. McWilliams. Quasi-monotone advection schemes based on explicit locally adaptive dissipation. Mon. Wea. Rev., 126):1541-1580, 1998.

Shchepetkin, A. F. and J. C. McWilliams. The regional oceanic modeling system (ROMS): a split-explicit, free-surface, topography-following-coordinate oceanic model. Ocean Modelling, 9:347-404, 2005.

Smith, R., M. Maltrud, F. Bryan, and M. Hetcht. Numerical simulations of the north atlantic ocean at 1/10deg. J. Phys. Oceanogr., 30:1532-1561, 2000.

Umlauf, L., H. Burchard, and K. Hutter. Extending the $k-\omega$ turbulence model towards oceanic applications. Ocean Modelling, 5:195-218, 2003.

Veneziani, M., A. Griffa, A. Reynolds, Z. Garraffo, and E. Chassignet. Parameterizations of lagrangian spin statistics and particle dispersion in presence of coherent vortices. J. Mar. Res., 63:1057-1083, 2005.

Waugh, D. W., E. R. Abraham, and M. M. Bowen. Spatial variations of stirring in the surface ocean: A case study of the Tasman Sea. J. Phys. Oceanogr., 36:526-542, Mar 2006.

Weiss, J. The dynamics of enstrophy transfer in 2-Dimensional hydrodynamics. Physica D, 48(2-3):273-294, MAR 1991. ISSN 0167-2789. 

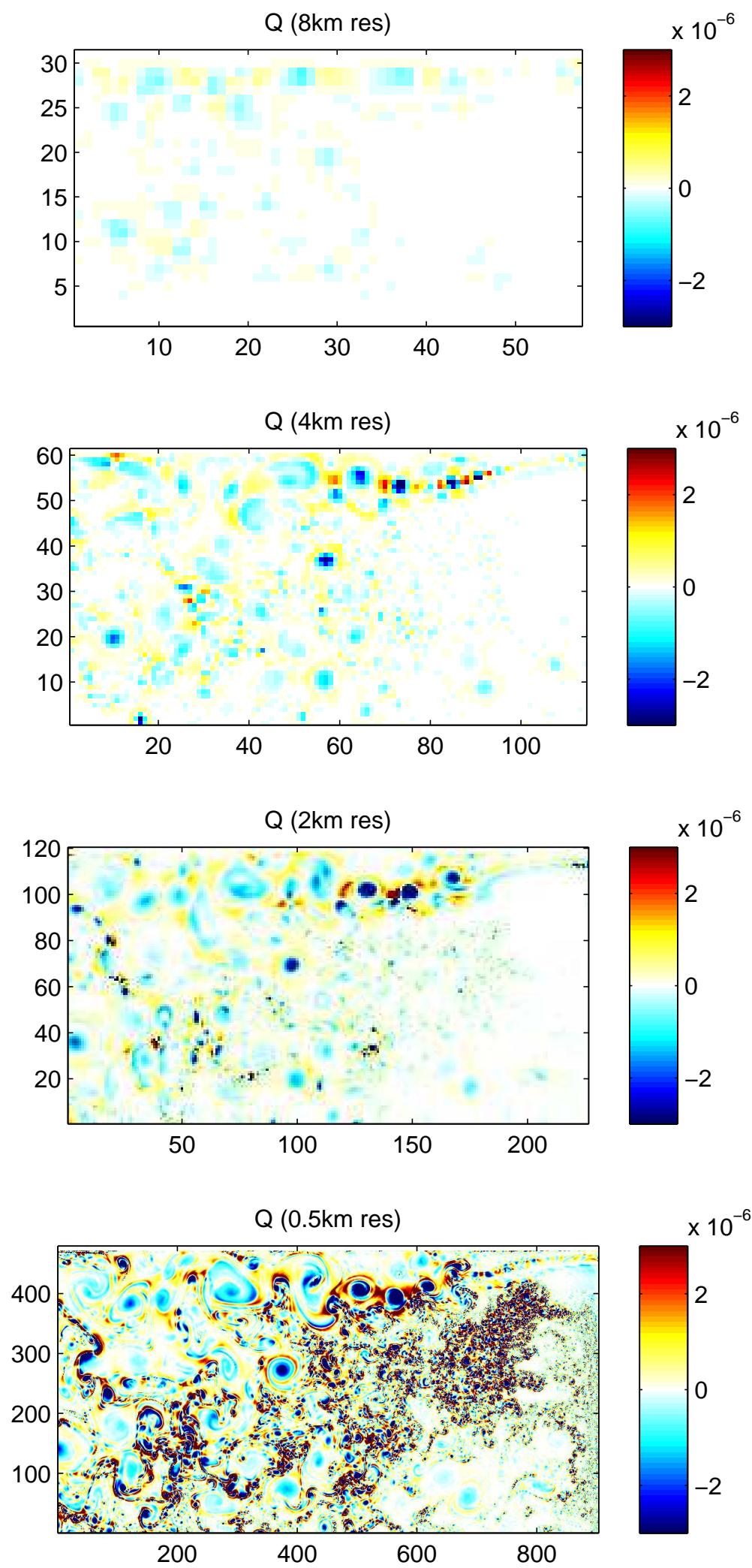

Figure 9: Snapshots of the Okubo-Weiss partition of the surface flow for increasingly finer horizontal resolution $(8,4,2$ and $0.5 \mathrm{~km})$. Units are in $\mathrm{sec}^{-2}$. 

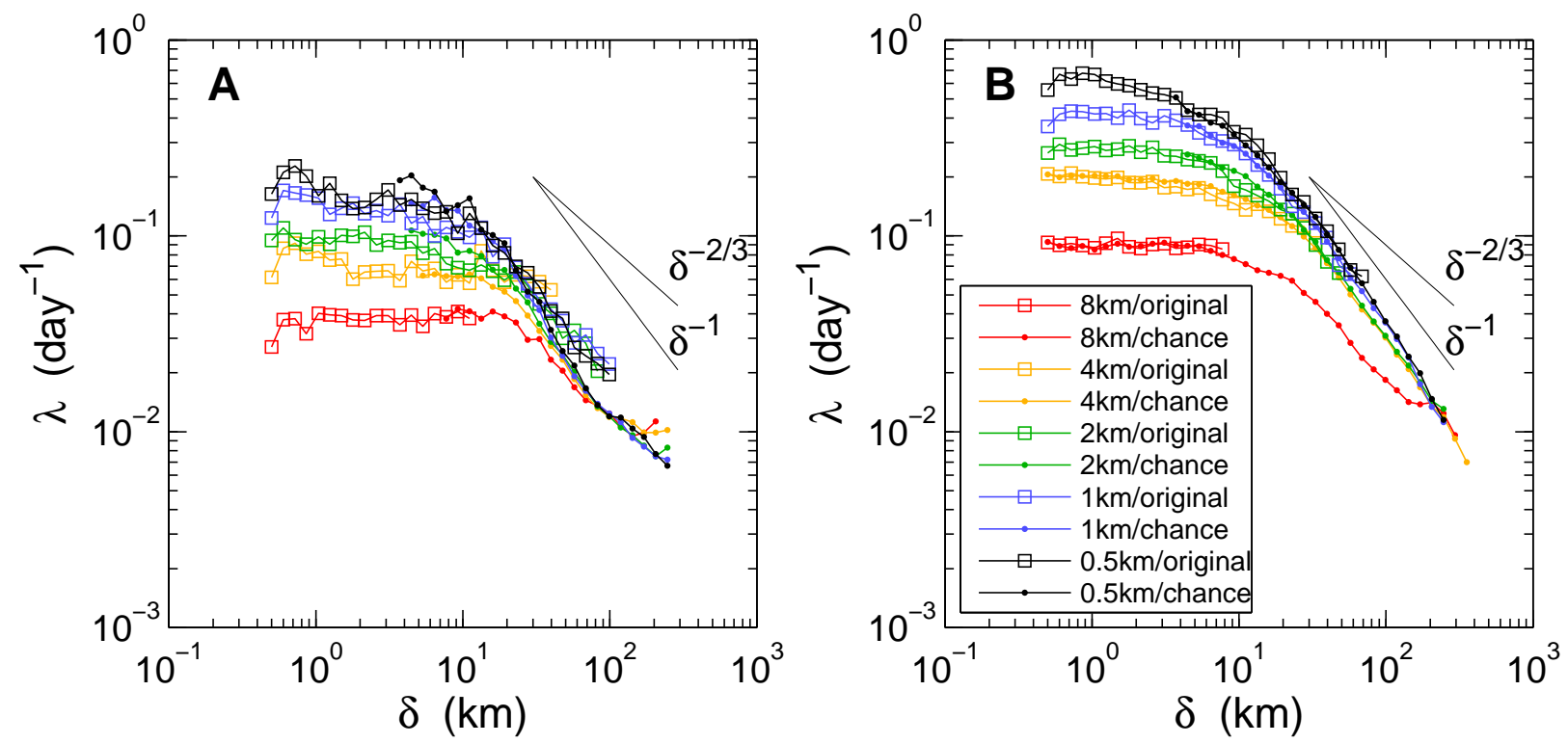

Figure 10: FSLE vs length-scale $\delta$ computed using (a) the first-crossing method and (b) the fastest-crossing method for spatial resolutions of $8 \mathrm{~km}$ (red), $4 \mathrm{~km}$ (yellow), $2 \mathrm{~km}$ (green), $1 \mathrm{~km}$ (blue) and $0.5 \mathrm{~km}$ (black). Lines marked with squares and dots indicate sampling with original and chance pairs, respectively. Slope corresponding to Richardson $\left(\delta^{-2 / 3}\right)$ and ballistic $\left(\delta^{-1}\right)$ laws are marked for convenience.
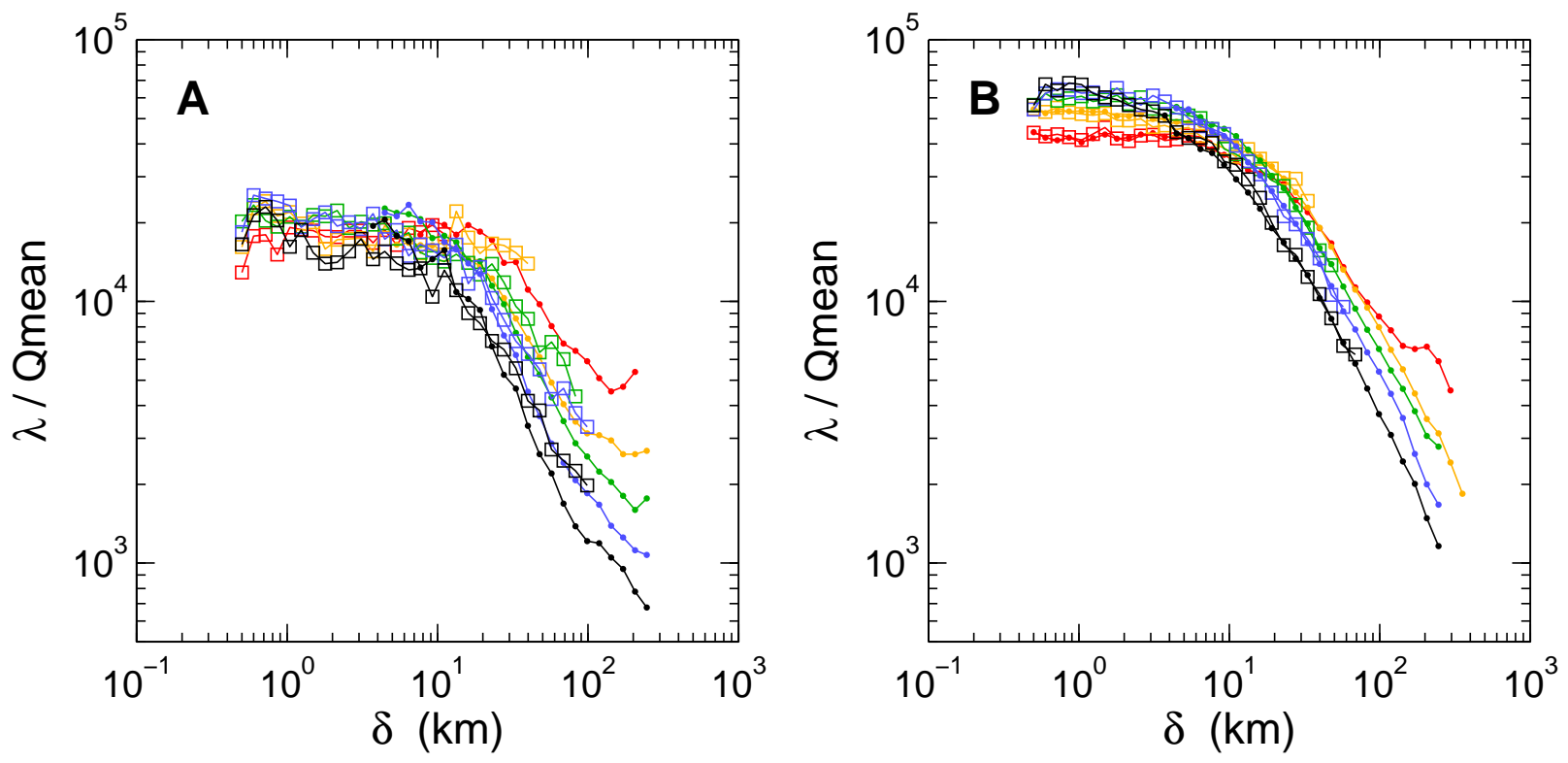

Figure 11: Same as Fig. 10, but after scaling $\lambda$ with $\bar{Q}$. 



Figure 12: (a) Snapshot of the HYCOM velocity field showing the Gulf Stream and meso-scale eddies as the main flow features. The color shows $\sqrt{u^{2}+v^{2}}$ in $m s^{-1}$. (b) Subset of the four-month trajectories from 14,615 particles launched between Cape Hatteras and the Grand Banks. 

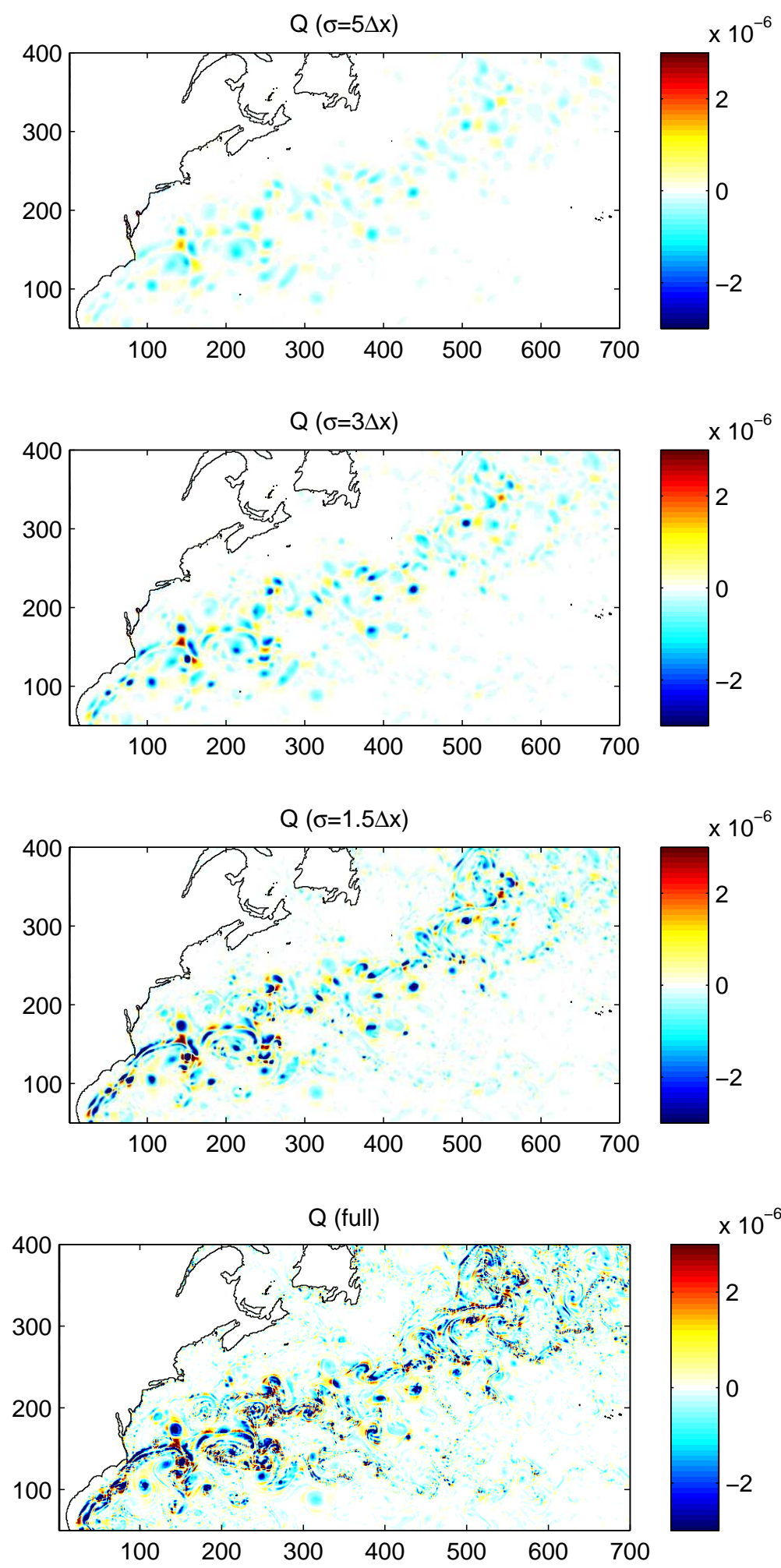

Figure 13: Okubo-Weiss snapshots of the HYCOM field shown in Fig. 12 subject to spatial smoothing with (a) $\sigma=5 \Delta x$, (b) $\sigma=3 \Delta x$, and (c) $\sigma=1.5 \Delta x$. Those from the original fields is shown in (d). Units are in $\sec ^{-2}$. 

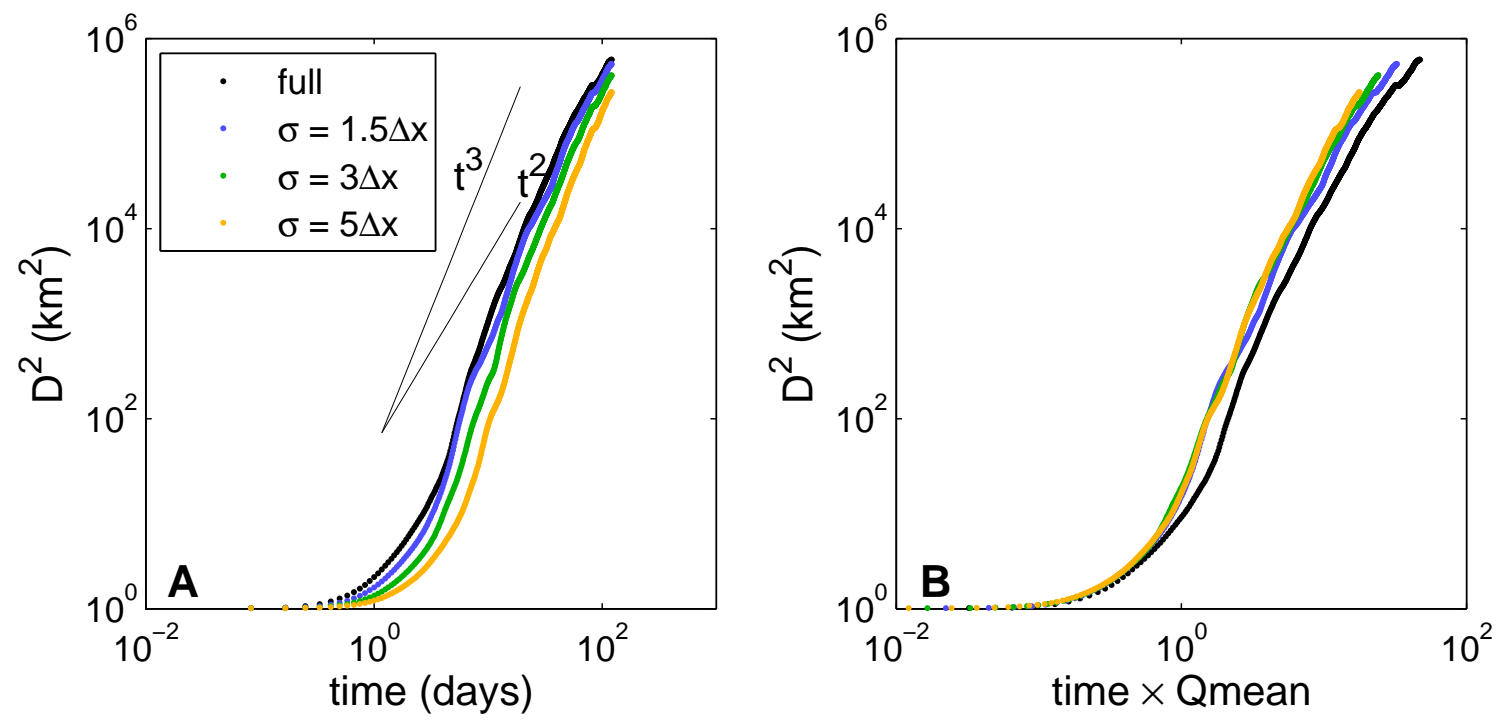

Figure 14: Relative dispersion versus time $D^{2}(t)$ computed from the five different cases, the full and spatially smoothed fields of HYCOM simulations.
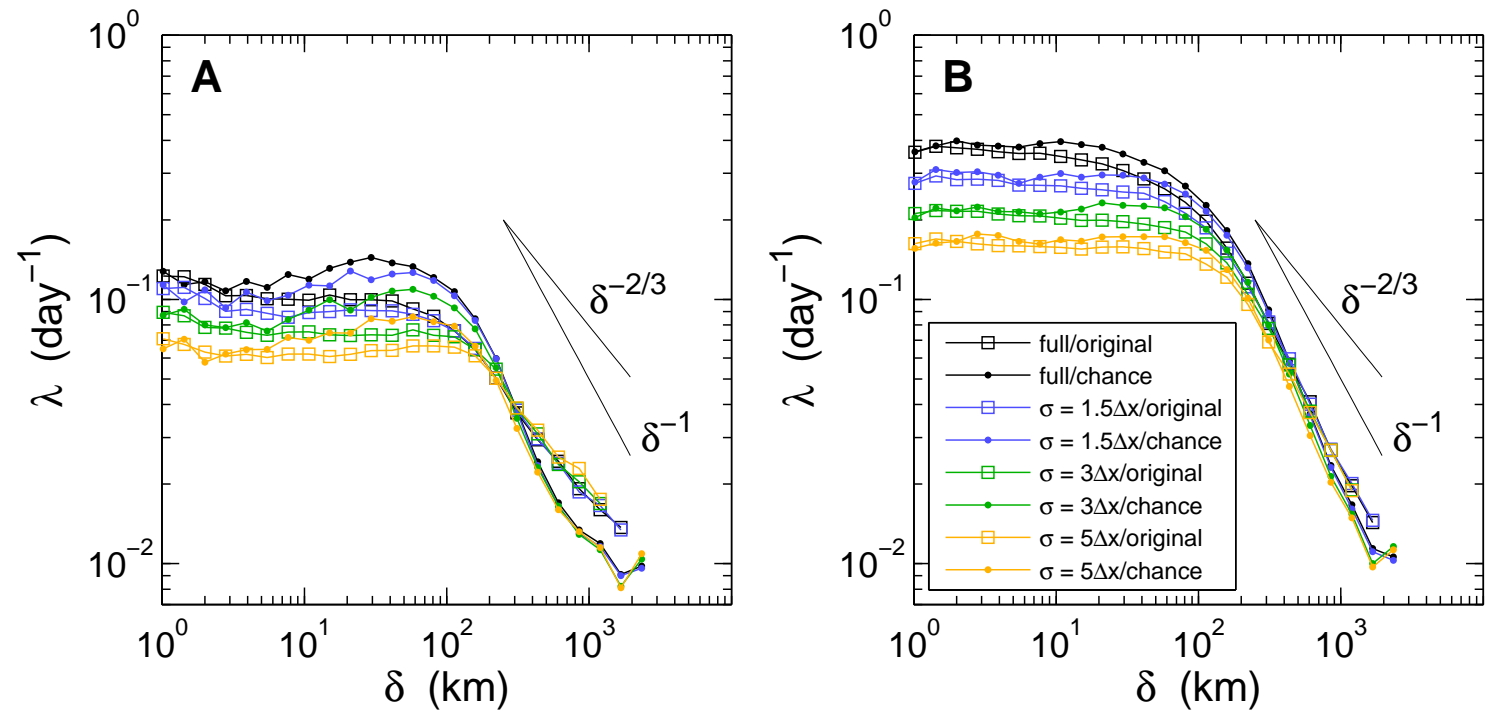

Figure 15: The FSLE $\lambda(\delta)$ computed using (a) the first-crossing method and (b) the fastest-crossing method for simulations from HYCOM output. Lines marked with squares and dots indicate sampling with original and chance pairs, respectively. 

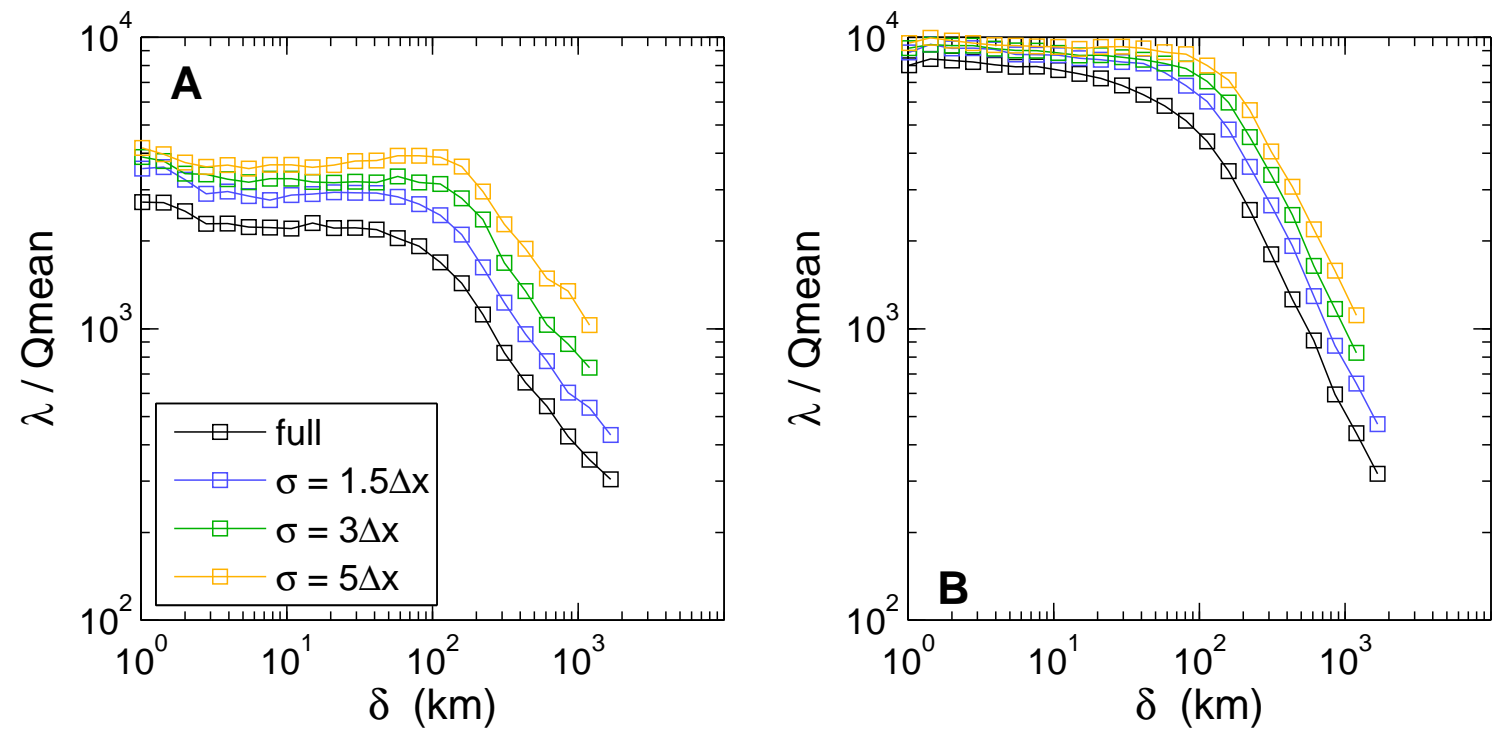

Figure 16: Same as Fig. 15, but after scaling $\lambda$ with $\bar{Q}$.

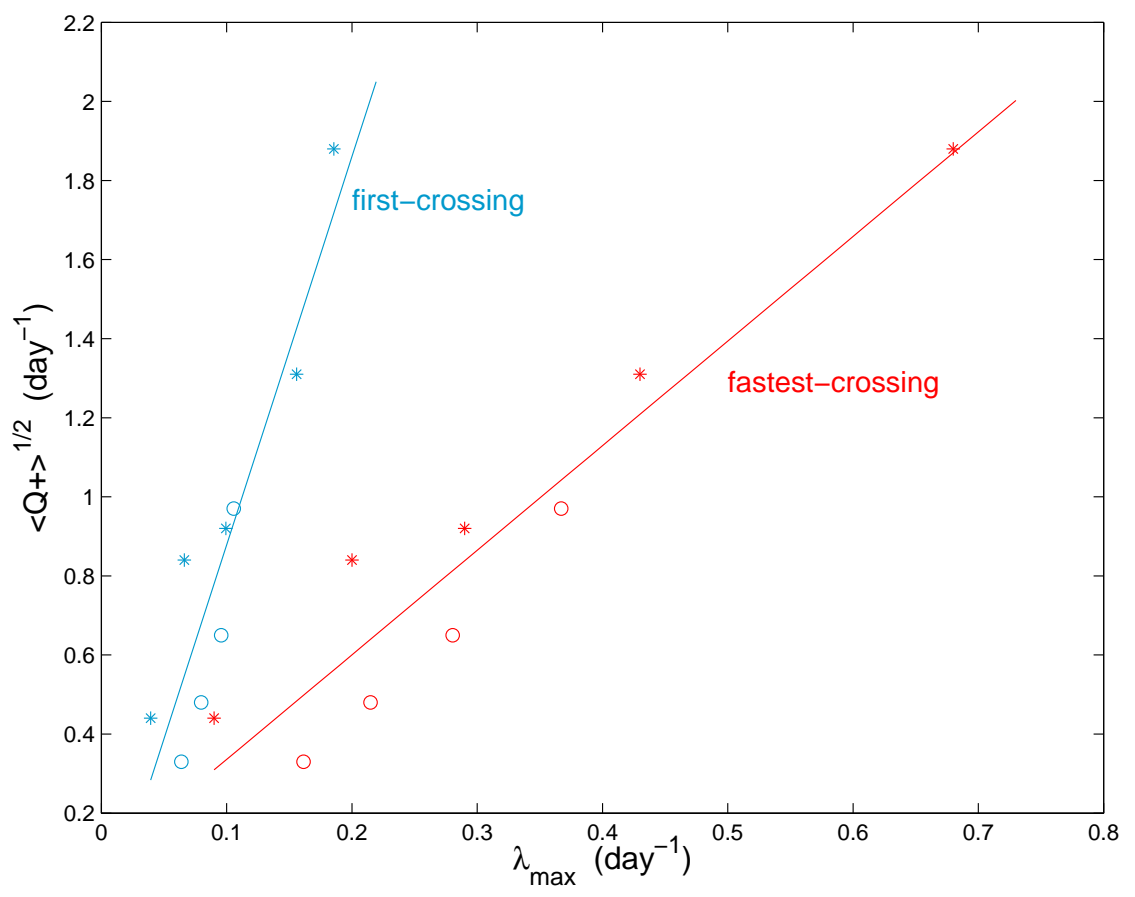

Figure 17: Scatter plot of the maximum value of $\lambda$ vs $\bar{Q}$ from ROMS (stars) and HYCOM (circles) for both first- and fastestcrossing FSLE computations. 